\title{
Self-similarity of Siegel disks and Hausdorff dimension of Julia sets
}

\author{
by
}

CURTIS T. MCMULLEN

Harvard University

Cambridge, MA, U.S.A.

\section{Contents}

1. Introduction . . . . . . . . . . . . . . . . . . . . . . . . . . . . . . . . . . . 247

2. Rotations of the circle . . . . . . . . . . . . . . 252

3. Visiting the critical point . . . . . . . . . . . . . 257

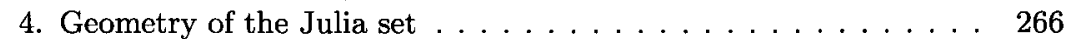

5. Universality . . . . . . . . . . . . . . . . . . . . . . . . . . . . . . . . . . . . . .

6. Renormalization . . . . . . . . . . . . . . . . 275

7. Self-similarity . . . . . . . . . . . . . . . . . . . . 280

8. The limiting dynamics . . . . . . . . . . . . . . . 283

A. Appendix: Promoting combinatorial equivalence to conjugacy . . 288

\section{Introduction}

Let $f: \mathbf{C} \rightarrow \mathbf{C}$ be the quadratic polynomial

$$
f(z)=e^{2 \pi i \theta} z+z^{2}
$$

where $\theta$ has continued fraction expansion

$$
\theta=\left[a_{1}, a_{2}, a_{3}, \ldots\right]=\frac{1}{a_{1}+\frac{1}{a_{2}+\frac{1}{a_{3}+\ldots}}} .
$$

Assume that $\theta$ is an irrational of bounded type, meaning $\sup a_{i}<\infty$. Then by a classical result of Siegel, the map $f$ is conformally conjugate to a rotation near the origin [Si].

Research partially supported by the NSF. 
The maximal domain $D$ on which this conjugacy takes place is the Siegel disk for $f$; it is the component of the interior of the filled Julia set $K(f)$ containing the origin.

Let $c_{0}=-\frac{1}{2} e^{2 \pi i \theta}$ denote the critical point of $f$, let $c_{i}=f^{i}\left(c_{0}\right)$, and let

$$
P(f)=\overline{\left\{c_{1}, c_{2}, c_{3}, \ldots\right\}}
$$

be the postcritical set. By work of Herman and Świątek, $\partial D=P(f)$ and $\partial D$ is a quasicircle passing through the critical point.

The behavior of $f$ is dominated by the transition between linear and nonlinear dynamics that occurs at the boundary of the Siegel disk. In this paper we study that transition and prove:

THEOREM 1.1. The Julia set $J(f)$ has Hausdorff dimension strictly less than two.

TheOREM 1.2. If $\theta$ is a quadratic irrational, then the boundary of the Siegel disk for $f$ is self-similar about the critical point.

Here is a more precise statement of the second theorem. Suppose that $\theta$ is a quadratic irrational; its continued fraction expansion is then preperiodic, satisfying $a_{n+s}=a_{n}$ for all $n \gg 0$. For simplicity assume that the period $s$ is even. Then we find that the periodicity of $\left\langle a_{n}\right\rangle$ allows one to construct a symmetry of $f$, namely a homeomorphism $\psi: \mathbf{C} \rightarrow \mathbf{C}$ satisfying

$$
\psi \circ f^{q_{n s}}(z)=f^{q_{n(s+1)}} \circ \psi(z)
$$

for $n \gg 0$ and $z$ near $c_{0}$, where $p_{n} / q_{n}=\left[a_{1}, \ldots, a_{n}\right]$. We show that $\psi$ stabilizes the Siegel disk and fixes the critical point; its complex derivative $\psi^{\prime}\left(c_{0}\right)$ exists; we have $0<\left|\psi^{\prime}\left(c_{0}\right)\right|<1$; and $\psi$ behaves like a $C^{1+\alpha}$-diffeomorphism near the critical point.

Since $\psi^{\prime}\left(c_{0}\right)$ is contracting, all features of $\partial D$ repeat at every scale, and the Siegel disk exhibits a self-similar shape ( $\$ 7)$. For example, the successive closest returns of the critical orbit $\left\langle c_{q_{n}}\right\rangle$ shadow geometric series converging to $c_{0}$.

The dynamical system generated by $f$ is also self-similar: when suitably rescaled, the maps $\left\langle f^{q_{n s+i}}(z)\right\rangle$ converge exponentially fast to a self-similar family $\left\langle g_{i}(z)\right\rangle$ of commuting holomorphic branched coverings of the plane ( $\S 8)$. Conceptually this means that $f$ is attracted to a fixed point of renormalization.

For $\theta$ of bounded type, we also prove that a form of universality holds ( $\S 5$ ):

THEOREM 1.3. Let $g: U \rightarrow V$ be a quadratic-like map with a fixed point of multiplier $e^{2 \pi i \theta}$. Then there is a conjugacy between $f$ and $g$ which is $C^{1+\alpha}$ and conformal on the boundary of the Siegel disk.

This result shows that the fine-scale behavior of $f \mid \partial D$ depends only on the combinatorics of $f$, not on its explicit analytical form. For example, the Hausdorff dimension 
of the boundary of the Siegel disk for $g_{a}(z)=e^{2 \pi i \theta} z+z^{2}+a z^{3}$ is constant for small values of $a$.

In the course of the discussion, we also find $(\S 4)$ :

(1) The Julia set of $f$ is shallow (or porous): there is an $\varepsilon>0$ such that any $r$-ball in $\mathbf{C}$ contains an $\varepsilon r$-ball disjoint from $J(f)$. On the other hand:

(2) The critical point is a Lebesgue density point of the filled Julia set; in fact,

$$
\operatorname{area}\left(B\left(c_{0}, r\right)-K(f)\right)=O\left(r^{2+\alpha}\right)
$$

for some $\alpha>0$.

(3) The dynamical system $(\mathcal{F}(f), J(f))$ is uniformly twisting: at every scale near every point in the Julia set, one can find a degree-two branched covering $h: U \rightarrow V$ with bounded geometry, contained in the full dynamics $\left\langle f^{-i} \circ f^{j}\right\rangle$.

The golden mean. All these assertions apply to the golden mean Siegel disk, where $\theta=\frac{1}{2}(\sqrt{5}-1)=[1,1,1, \ldots]$; see Figure 1. The critical point is in the center of the picture, which depicts the filled Julia set in gray; the quasicircle bounding the Siegel disk is outlined in black at the lower left.

We also show that the boundary of the golden mean Siegel disk does not spiral about the critical point (Corollary 7.5). This means that any continuous branch of $\arg \left(z-c_{0}\right)$ defined along $\partial D-\left\{c_{0}\right\}$ is bounded. The same result holds whenever the continued fraction expansion of $\theta$ has odd period.

Empirically, the golden mean Siegel disk has the stronger property that $D$ contains a triangle with one vertex at $c_{0}$.

Sources of rigidity. Convergence of the renormalized dynamics for the golden mean Siegel disk and its cousins may at first sight appear unrelated to considerations such as the dimension of the Julia set. In fact a principal goal of this paper is to give an explanation for convergence of renormalization directly in terms of the geometry of the filled Julia set $K(f)$ and its boundary $J(f)$. Here is a sketch of the argument.

Since $\partial D$ is a quasicircle, there is definite space inside $K(f)$ near every $z \in \partial D$. The dynamics propagates this space throughout the Julia set, and we conclude that $\operatorname{dim} J(f)<2$ (Theorem 1.1).

The picture is enhanced by the observation that the critical point (indeed every $z \in \partial D$ ) is a Lebesgue density point of $K(f)$. To see this, note that points just outside the Siegel disk must visit the critical point many times before they can escape to infinity. On each visit, there is a definite chance of landing in the preimage $D^{\prime}$ of $D$. Thus a random point close to $\partial D$ has a high probability of eventually landing in $D^{\prime}$, so the density of $K(f)$ tends to one. (The study of $f$ near $\partial D$ is facilitated by working in the 

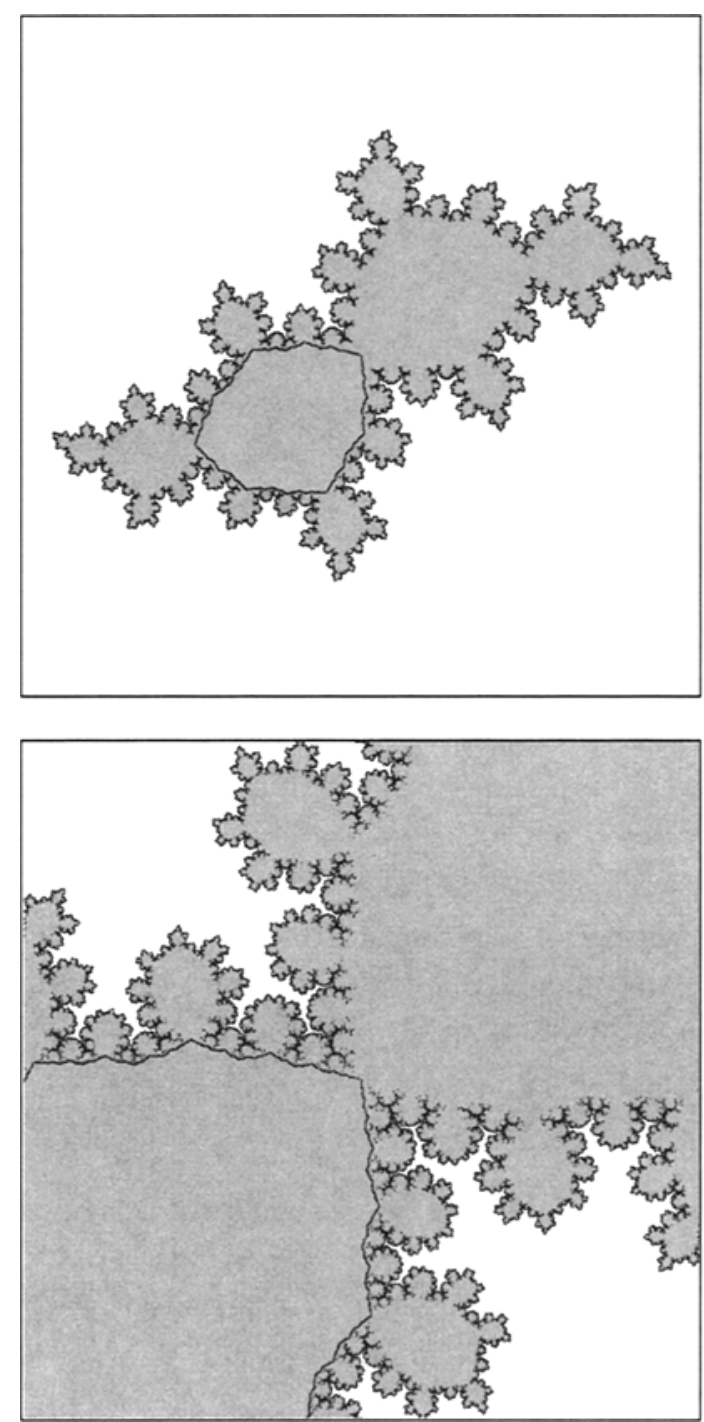

Fig. 1. The golden mean Siegel disk and blowup around the critical point

linear coordinate system, a quasiconformal chart in which $D$ is the unit disk, $f \mid D$ is a rigid rotation, and the iterates of $f$ are uniformly quasiregular.)

Next we discuss universality. Consider a quadratic-like map $g(z)$ with a fixed point of multiplier $e^{2 \pi i \theta}$. By a basic result of Douady and Hubbard, $g=\phi \circ f \circ \phi^{-1}$, where $\phi$ is conformal on $K(f)$. Measure-theoretically, the filled Julia set is sufficiently dense near $z \in \partial D$ that the conformal behavior of $\phi$ dominates; we conclude that $\phi^{\prime}(z)$ exists and that $\phi \mid \partial D$ is $C^{1+\alpha}$ for some $\alpha>0$. 


\begin{tabular}{|c|c|}
\hline \multicolumn{2}{|c|}{ Parallels } \\
\hline $\begin{array}{l}\text { Infinitely renormalizable map } \\
\qquad f(z)=z^{2}+c, c \in \mathbf{R}\end{array}$ & $\begin{array}{c}\text { Siegel linearizable map } \\
f(z)=e^{2 \pi i \theta} z+z^{2}, \theta \in \mathbf{R} / \mathbf{Z}\end{array}$ \\
\hline Tuning invariant & Continued fraction \\
\hline$c=s_{1} * s_{2} * s_{3} \ldots$ & $\theta=\left[a_{1}, a_{2}, \ldots\right]$ \\
\hline Bounded combinatorics & Bounded type \\
\hline$P(f)=$ quasi-Cantor set & $P(f)=$ quasicircle \\
\hline Quadratic-like map & Holomorphic pair \\
\hline Feigenbaum polynomial & Golden mean polynomia \\
\hline$\left(\mathbf{Z}_{2}, x+1\right)$ & $\left(S^{1}, x+\theta\right)$ \\
\hline$f^{n}, n=1,2,4,8,16, \ldots$ & $f^{n}, n=1,2,3,5,8,13, \ldots$ \\
\hline \multicolumn{2}{|c|}{$(\mathcal{F}(f), J(f))$ is uniformly twisting } \\
\hline \multicolumn{2}{|c|}{ The critical point of $f$ is a deep point of $K(f)$} \\
\hline \multicolumn{2}{|c|}{ Conjugacies are $C^{1+\alpha}$ on $P(f)$} \\
\hline
\end{tabular}

Table 2

A similar argument provides smoothness of the conjugating map $\psi$ between two renormalizations of $f$. Thus $\partial D$ is self-similar when $\theta$ is a quadratic irrational (Theorem 1.2). To construct these renormalizations, which are variants of de Faria's commuting pairs $[\mathrm{F}]$, we use Petersen's result on local connectivity of $J(f)[\mathrm{Pe}]$.

Finally we describe the limiting dynamics. By smoothness of $\psi$ and a harmonic measure argument, one finds that for $i \in \mathbf{Z}$, the rescaled first return maps $f^{q_{n s+i}}$ converge exponentially fast as $n \rightarrow \infty$. Their limit $g_{i}: W_{i} \rightarrow \mathbf{C}$ is an infinite-sheeted branched covering of the plane, with $\partial W_{i}$ as its natural boundary; moreover, $g_{i}$ can be expressed as a countable union of proper maps. We have $g_{i+s}=A \circ g_{i} \circ A^{-1}$, where $A$ is the linearization of $\psi$; thus the limiting dynamical system is a fixed point of renormalization. By universality, the same limit arises starting with any quadratic-like map with a fixed point of multiplier $e^{2 \pi i \theta}$.

Notes and references. Various forms of the universality and self-similarity results we establish here were conjectured and observed numerically more than a decade ago in the physics literature [MN], [Wi]. Petersen proved that the Lebesgue area of $J(f)$ is zero and that $J(f)$ is locally connected when $\theta$ has bounded type [Pe]. On the other hand, if $\theta=\left[a_{1}, a_{2}, \ldots\right]$ and $a_{n} \rightarrow \infty$ sufficiently fast, then there is no Siegel disk, $J(f)$ is not locally connected and Shishikura shows that the Hausdorff dimension of $J(f)$ is two [Sh]. The regime of unbounded type still presents many mysteries. 
This paper is a sequel to [Mc4], which develops the theme of rigidity and inflexibility in conformal dynamics and hyperbolic geometry. Most of our results for the golden mean Siegel disk have parallels for the Feigenbaum polynomial $f(z)=z^{2}+c_{F}$ (the unique real quadratic polynomial such that $f^{n}(z)$ is renormalizable for $\left.n=1,2,4,8, \ldots\right)$. These parallel results are established in [Mc4] and are summarized in Table 2.

A critical circle map is a smooth homeomorphism $f: S^{1} \rightarrow S^{1}$ with a single critical point. The self-similarity of Siegel disks is also related to convergence of renormalization for critical circle maps, studied in $[\mathrm{F}]$ and $[\mathrm{FM}]$.

I would like to thank de Faria, de Melo and Petersen for useful conversations about their work, and Petersen and the referee for helpful suggestions and corrections.

\section{Rotations of the circle}

Let $S^{1}=\mathbf{R} / \mathbf{Z}$, let $\theta \in(0,1)$ be an irrational number with continued fraction expansion $\theta=\left[a_{1}, a_{2}, \ldots\right]$, and let $F: S^{1} \rightarrow S^{1}$ be the rotation $F(x)=x+\theta$. In this section we will briefly summarize some relations between the dynamics of $F$ and the continued fraction expansion of $\theta$. See [HW, Chapter X] for a detailed treatment of continued fractions.

For $x \in S^{1}$ let $\{x\}$ denote the unique real number representing $x$ in $\left(-\frac{1}{2}, \frac{1}{2}\right]$, and let $\|x\|=|\{x\}|$ denote the distance from $x$ to 0 .

Let

$$
\frac{p_{n}}{q_{n}}=\left[a_{1}, \ldots, a_{n}\right]
$$

denote the rational approximations to $\theta$ (in lowest terms) obtained by truncating its continued fraction expansion.

The points $q_{n} \theta, n=1,2, \ldots$, are successive closest returns of the origin to itself under rotation by $\theta$. That is,

$$
\theta>\left\|q_{1} \theta\right\|>\left\|q_{2} \theta\right\|>\left\|q_{3} \theta\right\|>\ldots
$$

and the $q_{n}$ 's can be defined inductively as the smallest integers so the above inequalities hold.

The closest returns occur alternately to the right and left of the origin. In fact, $(-1)^{n}\left\{q_{n} \theta\right\}>0$, and the fractional part is given by

$$
\left\{q_{n} \theta\right\}=q_{n} \theta-p_{n}
$$

The $a_{n}$ are related to $q_{n}$ and $p_{n}$ by the recursive formulas

$$
\begin{aligned}
& p_{n}=a_{n} p_{n-1}+p_{n-2}, \\
& q_{n}=a_{n} q_{n-1}+q_{n-2},
\end{aligned}
$$




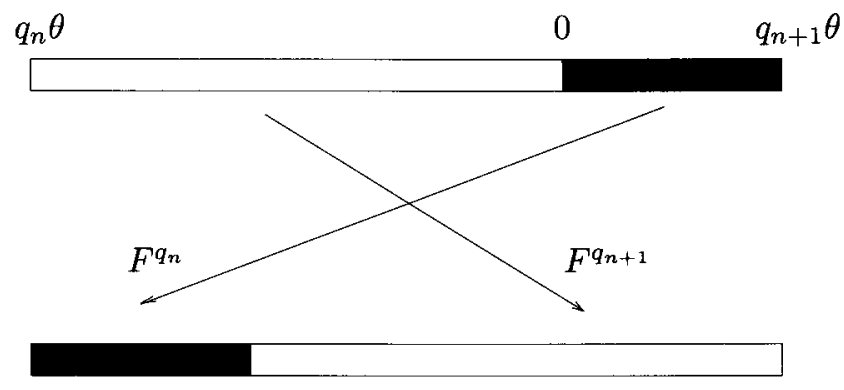

Fig. 3. Renormalized rotation

where $p_{-1} / q_{-1}=1 / 0$ and $p_{0} / q_{0}=0 / 1$. Since $a_{n} \geqslant 1$, this recursion shows that $q_{n}$ grows at least as fast as the Fibonacci sequence $1,2,3,5,8, \ldots$, and therefore $q_{n} \rightarrow \infty$ at least as fast as the $n$th power of the golden mean.

First return maps. For $x, x^{\prime} \in S^{1}$ with $x \neq \frac{1}{2}+x^{\prime}$, let $\left[x, x^{\prime}\right]$ denote the shorter interval bounded by these points. (Note that $\left[x, x^{\prime}\right]=\left[x^{\prime}, x\right]$.)

Let $I_{n}=\left[q_{n} \theta, q_{n+1} \theta\right]$ be the interval bounded by two successive closest returns to the origin. Note that 0 lies in $I_{n}$. Let us consider the dynamics induced by the first return map on $I_{n}$; that is, for $x \in I_{n}$ we consider the least $i>0$ such that $F^{i}(x) \in I_{n}$. The result is the renormalized dynamical system generated by the two transformations:

$$
\begin{gathered}
F^{q_{n}}:\left[0, q_{n+1} \theta\right] \rightarrow\left[q_{n} \theta, x_{n}\right], \\
F^{q_{n+1}}:\left[q_{n} \theta, 0\right] \rightarrow\left[x_{n}, q_{n+1} \theta\right],
\end{gathered}
$$

where $x_{n}=\left(q_{n}+q_{n+1}\right) \theta$. This pair of transformations is an interval exchange map; see Figure 3 .

The orbit of every point $x \in S^{1}$ is dense, so $x$ eventually enters $I_{n}$. How long does it take to do so? We claim that

$$
F^{i}(x)=x+i \theta \in I_{n} \quad \text { for some } i, 0 \leqslant i<q_{n+1} .
$$

To see this, first choose $j<0$ such that $F^{j}(x) \in I_{n}$. The generators of the renormalized dynamics $\left\langle F^{q_{n}}, F^{q_{n+1}}\right\rangle$ map $I_{n}$ onto itself, so we may apply them one at a time to replace $j$ by $j+q_{n}$ or $j+q_{n+1}$ while keeping $F^{j}(x) \in I_{n}$. When $j$ first becomes nonnegative it lies in the range $\left[0, q_{n+1}\right)$ as claimed.

Now let $J_{n}$ be the interval centered in $I_{n}$ with length $\left|J_{n}\right|=\left|I_{n}\right|-\left\|q_{n+1} \theta\right\|$. In other words, $J_{n}$ excludes the outer halves of the two short black intervals appearing in Figure 3.

We claim that

$$
F^{i}(x)=x+i \theta \in J_{n} \quad \text { for some } i, 0 \leqslant i<q_{n}+q_{n+1} .
$$


To find $F^{i}(x)$ with this property, begin by choosing $j$ with $0<j<q_{n+1}$ and $F^{j}(x) \in I_{n}$. If $F^{j}(x) \in J_{n}$ we are done. Otherwise $F^{j}(x)$ lies near one of the endpoints of $I_{n}$. If $F^{j}(x)$ is closer to $q_{n+1} \theta$, then $F^{j+q_{n}}(x) \in J_{n}$, and since

$$
j+q_{n}<q_{n+1}+q_{n}
$$

we are done. Finally if $F^{j}(x)$ is closer to $q_{n} \theta$, then both $x+\left(j+q_{n+1}\right) \theta$ and $x+\left(j-q_{n}\right) \theta$ lie in $J_{n}$; the first of these works if $j<q_{n}$, and the second if $j \geqslant q_{n}$.

Univalent branches. We will eventually apply these results to the quadratic polynomial $f$, using the fact that $\left(F, S^{1}\right)$ is topologically conjugate to $f$ on the boundary of the Siegel disk. In this application $0 \in S^{1}$ will correspond to the critical point of $f$, and we will be interested in constructing a univalent restriction of $f^{i}$ sending $x$ into $I_{n}$. Now the critical values of $f^{i}$ correspond to $\theta, 2 \theta, \ldots, i \theta$; since $i<q_{n}+q_{n+1}$, there are no critical values in the interior of the interval $I_{n}$, and therefore $f^{-i} \mid \operatorname{int}\left(I_{n}\right)$ has univalent branches. The fact that $f^{i}(x)$ lands in the strictly smaller interval $J_{n}$ provides Koebe space for the inverse univalent map.

Bounded type. The orbits of a bounded type rotation are especially evenly distributed. The following are equivalent characterizations of an irrational $\theta$ of bounded type:

(1) The partial quotients $a_{n}$ of $\theta$ are bounded.

(2) The number $\theta$ is Diophantine of exponent two; that is, there is a $C>0$ such that

$$
\left|\theta-\frac{p}{q}\right|>\frac{C}{q^{2}}
$$

for every rational $p / q$.

(3) The denominators $q_{n}$ increase no faster than a geometric series; that is, $q_{n}<C^{n}$ for some $C>1$.

(4) Adjacent closest returns of $F^{i}(0)$ are approximately evenly spaced; that is,

$$
\left\|q_{n} \theta\right\|>\left\|q_{n+1} \theta\right\|>C\left\|q_{n} \theta\right\|
$$

for some $C>0$.

(5) For any $k>0$, the orbit $F^{i}(0), i=1, \ldots, k$, cuts the circle into intervals of approximately the same size; that is, the ratio of the longest to the shortest is bounded above independent of $k$.

(6) We have $\left|J_{n}\right| /\left|I_{n}\right|>C>0$ for all $n$.

The constants appearing above can be chosen to depend only on sup $a_{n}$. 
Preperiodic continued fractions. Finally we describe the self-similar behavior of rotations where the continued fraction of $\theta$ is preperiodic.

THEOREM 2.1. Let $\theta=\left[a_{1}, a_{2}, \ldots\right]$, where $a_{n+s}=a_{n}$ for $n \geqslant N$. Then there is a real number $\gamma \in(0,1)$ such that

$$
\left\{q_{n+s} \theta\right\}=(-1)^{s} \gamma\left\{q_{n} \theta\right\}
$$

for $n \geqslant N$.

Proof. For any continued fraction, one has

$$
A_{n}=\left(\begin{array}{cc}
a_{n} & -1 \\
-1 & 0
\end{array}\right) \ldots\left(\begin{array}{cc}
a_{1} & -1 \\
-1 & 0
\end{array}\right)=\left(\begin{array}{cc}
q_{n} & -p_{n} \\
-q_{n-1} & p_{n-1}
\end{array}\right)
$$

by (2.2). In addition, it is easy to prove by induction that

$$
A_{n}\left(\begin{array}{c}
\theta \\
1
\end{array}\right)=(-1)^{n} \theta_{1} \theta_{2} \ldots \theta_{n}\left(\begin{array}{c}
\theta_{n+1} \\
1
\end{array}\right)
$$

where $\theta_{i}=\left[a_{i}, a_{i+1}, a_{i+2}, \ldots\right]$.

Now suppose that the continued fraction for $\theta$ is periodic with period $s$. Then

$$
A_{n+s}\left(\begin{array}{l}
\theta \\
1
\end{array}\right)=A_{n} A_{s}\left(\begin{array}{l}
\theta \\
1
\end{array}\right)=(-1)^{s} \gamma A_{n}\left(\begin{array}{l}
\theta \\
1
\end{array}\right)
$$

where $\gamma=\theta_{1} \theta_{2} \ldots \theta_{s}$. Equivalently,

$$
\left(\begin{array}{cc}
q_{n+s} & -p_{n+s} \\
-q_{n+s-1} & p_{n+s-1}
\end{array}\right)\left(\begin{array}{l}
\theta \\
1
\end{array}\right)=(-1)^{s} \gamma\left(\begin{array}{cc}
q_{n} & -p_{n} \\
-q_{n-1} & p_{n-1}
\end{array}\right)\left(\begin{array}{l}
\theta \\
1
\end{array}\right)
$$

By (2.1), $\left\{q_{n} \theta\right\}=q_{n} \theta-p_{n}$, so the theorem follows by multiplying through above.

The preperiodic case is similar.

Example. Let $\theta=[1,1,1, \ldots]=\frac{1}{2}(\sqrt{5}-1)$ be the golden mean. Then $\gamma=\theta,\left\langle q_{1}, q_{2}, \ldots\right\rangle=$ $\langle 1,2,3,5,8,13, \ldots\rangle$, and we have $\left\{q_{n} \theta\right\}=(-1)^{n} \theta^{n+1}$. In this example (or any other with period one) the closest returns occur along a geometric series.

To obtain a more complete dynamical picture, for $n \geqslant 3$ define $r_{n}=q_{n-1}-q_{n}, L_{n}=$ $\left[r_{n+1} \theta, r_{n} \theta\right]$, and define the extended renormalization $\mathcal{R}_{n}(F)$ to be the pair of mappings

$$
\begin{gathered}
F^{q_{n}}:\left[0, r_{n} \theta\right] \rightarrow\left[q_{n} \theta, q_{n-1} \theta\right] \subset L_{n}, \\
F^{q_{n+1}}:\left[r_{n+1} \theta, 0\right] \rightarrow\left[q_{n} \theta, q_{n+1} \theta\right] \subset L_{n} .
\end{gathered}
$$

Then $L_{n} \supset I_{n}$ and the maps above extend the interval exchange dynamics defined by (2.3). 
The choice of the domains for $F^{q_{n}}$ and $F^{q_{n+1}}$ is dictated by branching considerations, again under the topological conjugacy between $F$ and the quadratic polynomial $f$ acting on the boundary of its Siegel disk. If $x=0$ corresponds to the critical point of $f$, then the critical values of $f^{i}$ on the boundary of the Siegel disk correspond to $\{\theta, 2 \theta, \ldots, i \theta\} \subset S^{1}$. Thus the interval $\left[q_{n} \theta, q_{n-1} \theta\right]$ comprising the range of $F^{q_{n}}$ above is the maximal interval containing $x=0$ and with no critical values in its interior. Similarly, the domain $\left[0, r_{n} \theta\right]$ is a maximal interval whose interior is disjoint from the critical points of $F^{q_{n}}$, and the same considerations dictate the domain and range of $F^{q_{n+1}}$.

COROLlARY 2.2. The similarity $x \mapsto(-1)^{s} \gamma x$ sends $L_{n}$ to $L_{n+s}$ and conjugates $\mathcal{R}_{n}(F)$ to $\mathcal{R}_{n+s}(F)$, for all $n \geqslant \max (N, 3)$.

Proof. This follows from the theorem and the fact that $x \mapsto \alpha x$ conjugates rotation by $t$ to rotation by $\alpha t$.

The functional equation. The renormalization theory has a nice description on the universal cover of the circle. Let $T \cong \mathbf{R}$ be the group of translations of the real line, let $V=\operatorname{Hom}\left(\mathbf{Z}^{2}, T\right) \cong \mathbf{R}^{2}$ and let $\varrho_{\theta} \in V$ be given by $\varrho_{\theta}(i, j)=i \theta+j$. Then $\varrho_{\theta}$ represents the combined dynamics of the rotation $x \mapsto x+\theta$ and the deck transformation $x \mapsto x+1$ acting on the universal cover of $S^{1}=\mathbf{R} / \mathbf{Z}$.

Let $G \cong \mathbf{R}^{*}$ be the group of similarities $x \mapsto \alpha x$, and let $\bar{V}=V / / G \cong \mathbf{R} \mathbf{P}^{1}$ be the quotient of the nontrivial representations under conjugation by $G$. Then $G L_{2}(\mathbf{Z})=\operatorname{Aut}\left(\mathbf{Z}^{2}\right)$ acts on $\bar{V}$ by $[\varrho] \mapsto\left[\varrho \circ A^{-1}\right]$; this agrees with its usual action on $\mathbf{R P}^{1}$ by fractional linear transformations.

A representation $[\varrho]$ is fixed by $A$ if and only if there is a similarity $\alpha \in G$ such that

$$
\varrho \circ A^{-1}=\alpha \varrho \alpha^{-1}
$$

This functional equation says that the renormalized dynamical system $\varrho^{\circ} A^{-1}$ is just a rescaling of the original dynamical system $\varrho$. It can be compared to the CvitanovićFeigenbaum equation

$$
F^{p}(z)=\alpha^{-1} F(\alpha z)
$$

for unimodal maps, and the fixed-point equation

$$
\varrho^{\circ} \psi_{*}^{-1}=\alpha \varrho \alpha^{-1}
$$

for a hyperbolic 3-manifold which fibers over the circle, discussed in [Mc4].

THEOREM 2.3. Let $\theta$ be irrational. Then the following are equivalent: 
(1) $\left[\varrho_{\theta}\right]$ is fixed by an automorphism $A \neq \mathrm{id}$ in $G L_{2}(\mathbf{Z})$.

(2) $\theta$ is a quadratic irrational.

(3) The continued fraction expansion of $\theta$ is preperiodic.

Proof. If $A\left[\varrho_{\theta}\right]=\left[\varrho_{\theta}\right]$, then $(\theta, 1)$ is an eigenvector for $A$, so $\theta$ is quadratic over $\mathbf{Q}$. A quadratic irrational has a preperiodic continued fraction [HW, $\S 10.12]$, and by (2.4) a preperiodic continued fraction expansion gives a representation fixed by an automorphism.

\section{Visiting the critical point}

We now turn to the study of the polynomial $f(z)=e^{2 \pi i \theta} z+z^{2}$, where $\theta=\left[a_{1}, a_{2}, a_{3}, \ldots\right]$ is an irrational of bounded type.

The usual notations $A=O(B)$ and $A \asymp B$ mean $A<C B$ and $B / C<A<C B$, for an implicit constant $C$. In this section, the implicit constants depend only on $\theta$. (In fact, a detailed examination of the proofs shows that the bounds only depend on $\sup a_{n}$.)

To study $f$ we will use the Koebe distortion theorem, the Poincare metric and quasiconformal maps. See [Ah1], [Ah2], [LV] and [Mc3, Chapter 2] for background on these methods.

Metrics. We will use $d(x, y)$ to denote the Euclidean metric in the plane, and $d_{U}(x, y)$ for the hyperbolic metric on a region $U \subset \widehat{\mathbf{C}}$.

The Siegel disk. The filled Julia set $K(f)$ is defined by

$$
K(f)=\left\{z \in \mathbf{C}: \text { the forward orbit } f(z), f^{2}(z), f^{3}(z), \ldots \text { is bounded }\right\}
$$

it is bounded by the Julia set $J(f)$. The Siegel disk $D$ of $f$ is the component of the interior of $K(f)$ containing the origin. The critical point $c_{0}$ lies in $\partial D$ and $\partial D=P(f)$.

The central result which brings quasiconformal methods into play is

TheOREM 3.1 (Herman-Świątek). The Siegel disk of $f$ is bounded by a quasicircle.

See $[\mathrm{D}],[\mathrm{He}],[\mathrm{Sw}]$.

For a pointed disk $(U, u) \subset \mathbf{C}$, let

$$
\begin{aligned}
\operatorname{in}-\operatorname{radius}(U, u) & =\sup \{r: B(u, r) \subset U\}, \\
\operatorname{out}-\operatorname{radius}(U, u) & =\inf \{r: B(u, r) \supset U\} .
\end{aligned}
$$

The main result we will establish in this section is 


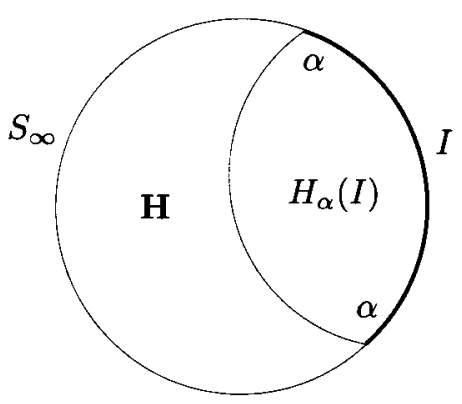

Fig. 4. The region $H_{\alpha}(I)$

THEOREM 3.2 (Nearby critical visits). For every $z \in J(f)$ and $r>0$, there is a univalent map between pointed disks of the form

$$
f^{i}:(U, y) \rightarrow\left(V, c_{0}\right), \quad i \geqslant 0
$$

such that in-radius $(U, y) \asymp r$, and $|y-z|=O(r)$.

Thus the geometry of $J(f)$ near the critical point is replicated with bounded distortion everywhere in the Julia set.

The linear coordinate. By the Herman-Świątek Theorem, the Riemann mapping from $(D, 0)$ to the unit disk $(\Delta, 0)$ extends to a quasiconformal mapping $\Phi:(\mathbf{C}, 0) \rightarrow(\mathbf{C}, 0)$. Let us normalize this mapping so that $\Phi\left(c_{0}\right)=1$. It is useful to work in both the coordinate system $z$ where $f$ is conformal, and the coordinate system $w=\Phi(z)$ where $f \mid D$ is linear. Since $\Phi$ is quasiconformal, many properties can be passed from one coordinate system to the other with a bounded change in constants.

For clarity we will denote the domain and range of $\Phi$ by $\mathbf{C}_{z}$ and $\mathbf{C}_{w}$ respectively. We refer to $w$ as the linear coordinate. In the $w$-coordinate system, $D$ is the unit disk, and $f: \mathbf{C}_{w} \rightarrow \mathbf{C}_{w}$ is a map whose iterates $f^{i}$ are uniformly quasiregular.

We identify the circle $S^{1}=\mathbf{R} / \mathbf{Z}$ with the unit circle in $\mathbf{C}_{w}$, via the map $t \mapsto \exp (2 \pi i t)$. For two points $x, x^{\prime} \in S^{1}$ we continue to use the notation $\left[x, x^{\prime}\right] \subset S^{1}$ to denote the shorter arc containing them.

Define $F: \mathbf{C}_{w} \rightarrow \mathbf{C}_{w}$ by $F(w)=e^{2 \pi i \theta} w$. Clearly $F^{i}=f^{i}$ on $D$. We will see that $F^{i}$ and $f^{i}$ tend to be close near $\partial D$ as well. To prove this, it is convenient to introduce some hyperbolic geometry.

Let $\mathbf{H}$ denote the hyperbolic plane, bounded by the circle $S_{\infty}^{1}$. For any closed interval $I \subset S_{\infty}^{1}$, let $H_{\alpha}(I) \subset \mathbf{H}$ be the region bounded by $I$ and a circular arc with endpoints $\partial I$, meeting $S_{\infty}^{1}$ in angle $\alpha$ (see Figure 4). For example, $H_{\pi / 2}(I)$ is the half-space formed by the convex hull of $I$. 
LEMMA 3.3. Let $g: H_{\alpha}(I) \rightarrow \mathbf{H}$ be a $K$-quasiconformal map which extends continuously to the identity on $I$. Then for all $x \in H_{\beta}(I), \beta<\alpha$, we have

$$
d_{\mathbf{H}}(g(x), x)<C,
$$

where $C$ depends only on $K, \beta$ and $\alpha$.

Proof. The image of $\partial H_{\beta}$ in $\mathbf{H}$ is a quasiarc, converging radially to $S_{\infty}^{1}$ because $\beta<\alpha$. This implies that $g \mid H_{\beta}$ can be extended to a $K^{\prime}$-quasiconformal map $h: \overline{\mathbf{H}} \rightarrow \overline{\mathbf{H}}$ with $h \mid S_{\infty}^{1}=$ id. Such a mapping moves points only a bounded hyperbolic distance.

Approximate rotations. Let

$$
\Omega=\mathbf{C}_{z}-\bar{D}
$$

denote the exterior of the Siegel disk in the plane. Let us say that $f^{i}: U \rightarrow V$ is an approximate rotation if $U$ and $V$ are disks in $\mathbf{C}_{z}, f^{i}$ is univalent and

$$
d_{\Omega}\left(f^{i}(x), F^{i}(x)\right)=O(1)
$$

for all $x \in \Omega \cap U$. Of course $f^{i}(x)=F^{i}(x)$ for $x \notin \Omega$, so this condition says that $f^{i}$ nearly matches up with the rotation $F^{i}$ throughout $U$.

THEOREM 3.4 (Approximate rotations). For any $r>0$ and $z \in P(f)$, there is an approximate rotation

$$
f^{i}:(U, y) \rightarrow\left(V, c_{0}\right), \quad i \geqslant 0,
$$

such that $[y, z] \subset U, \bmod (U-[y, z])>C>0$ and in-radius $(U, z) \asymp r$.

Remarks. (1) The proof will also show that $f^{j} \mid U$ is an approximate rotation for $j=1,2, \ldots, i$.

(2) The notation $\bmod (U-[y, z])$ denotes the conformal modulus of the annulus between the arc $[y, z] \subset P(f)$ and $\partial U$. The lower bound $C$ for this modulus depends only on $\theta$. It follows that the hyperbolic distance $d_{U}(y, z)=O(1)$.

(3) One also has in-radius $(U, y) \asymp r$.

(4) The theorem easily implies a slightly stronger statement, namely that $U$ and $V$ can be chosen as $K$-quasidisks, $K=O(1)$, with out-radius $(U, z) \asymp r$. To see this, replace $U$ by a large ball about $y$ in the hyperbolic metric on $U$, and apply the Koebe distortion theorem.

Proof. By general properties of quasiconformal mappings applied to the change of coordinates $\Phi: \mathbf{C}_{z} \rightarrow \mathbf{C}_{w}$, it is sufficient to prove the theorem in the linear coordinate $w$. For example, a quasiconformal map $\mathbf{H} \rightarrow \mathbf{H}$ is uniformly continuous in the hyperbolic metric, 


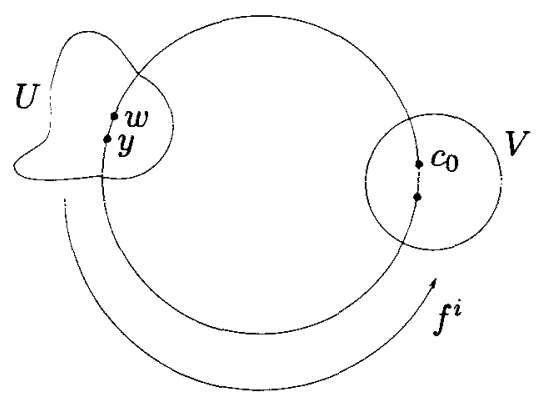

Fig. 5. An approximate rotation in the linear coordinate $w$

so it suffices to verify the approximate isometry condition $d_{\Omega}\left(f^{i}(x), F^{i}(x)\right)=O(1)$ using the hyperbolic metric coming from the $w$-coordinate. Similarly, if there are approximate isometries at every scale $r$ in the $w$-plane, then the same is true in the $z$-plane.

Letting $w=\Phi(z)$, we now proceed to prove the theorem at every scale $r$ about $w$. In this coordinate system, $f\left|S^{1}=F\right| S^{1}$ is rotation by angle $\theta$. Thus the results of $\S 2$ apply to the dynamics of $f$ on $S^{1}$.

The case $r \geqslant 1$ can be handled trivially by taking $y=c_{0}, U=V=B(w, 10 r)$ and $i=0$ (so $f^{i}=F^{i}=\mathrm{id}$ ). So assume $0<r<1$.

Choose $n$ such that $\left\|q_{n} \theta\right\| \asymp r$; this is possible because $\theta$ has bounded type. For convenience, also choose $n$ large enough that $\left\|q_{n} \theta\right\|<\frac{1}{8}$. Next choose the least $i \geqslant 0$ such that

$$
f^{i}(w) \in J_{n} \subset I_{n}=\left[q_{n} \theta, q_{n+1} \theta\right] \subset S^{1} .
$$

The critical point of $f$ is at angle zero on $S^{1}$, so $c_{0} \in J_{n}$.

As we saw in $\S 2, f^{i}$ has no critical values in $\operatorname{int}\left(I_{n}\right)$ because $i<q_{n}+q_{n+1}$. Let $V$ be the round disk orthogonal to $S^{1}$ and with $V \cap S^{1}=\operatorname{int}\left(I_{n}\right)$. Then there is a unique univalent branch

$$
f^{-i}: V \rightarrow U
$$

such that $f^{-i}\left(f^{i}(w)\right)=w$. See Figure 5 .

Now consider the region $H_{\alpha}\left(I_{n}\right) \subset \Omega$ determined by the hyperbolic geometry of $\Omega=$ $\mathbf{C}_{w}-\bar{\Delta}$. (This region is constructed by lifting $I_{n}$ to an interval on the boundary of the universal cover of $\Omega$, forming $H_{\alpha}\left(\tilde{I_{n}}\right)$ there and projecting down. We choose $\alpha$ small enough that the projection is an embedding.) One can find universal angles $\frac{1}{2} \pi<\beta<\alpha<\pi$ such that

$$
V \cap \Omega \subset H_{\beta}\left(I_{n}\right) \subset H_{\alpha}\left(I_{n}\right) .
$$

Indeed, when $\left|I_{n}\right|$ is small, we have $V \subset H_{\pi / 2+\varepsilon}\left(I_{n}\right)$.

Since the iterates of $f$ are uniformly quasiregular in the $w$-coordinate, the map $f^{-i}$ is $K$-quasiconformal on $H_{\alpha}\left(I_{n}\right)$ with $K=O(1)$. Furthermore, $F^{i}$ is conformal and 


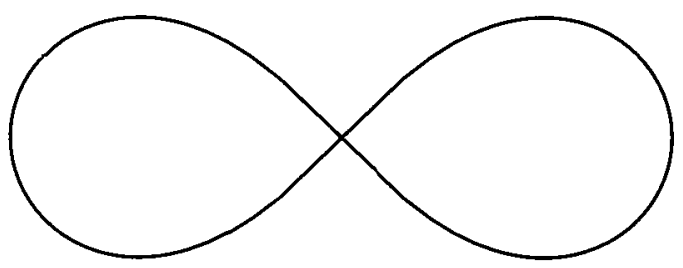

Fig. 6. A lemniscate models $\partial\left(D \cup D^{\prime}\right)$

$F^{i} \circ f^{-i}=$ id on $I_{n}$. Therefore Lemma 3.3 shows that $d_{\Omega}\left(F^{i}(x), f^{i}(x)\right)=O(1)$ for all $x \in U \cap \Omega$. In other words, $f^{i}: U \rightarrow V$ is an approximate isometry. By the same reasoning, $f^{j} \mid U$ is an approximate isometry for $0<j<i$.

Finally, setting $y=f^{-i}\left(c_{0}\right)$, we show that the annulus $U-[w, y]$ has definite modulus, and in-radius $(U, w) \asymp r$. To prove these statements, recall that $J_{n}$ is shorter than $I_{n}$ by a definite factor (because $\theta$ has bounded type). Thus the annulus $V-J_{n}$ has definite modulus. But

$$
\bmod (U-[w, y]) \geqslant K^{-1} \bmod \left(V-\left[f^{i}(w), c_{0}\right]\right) \geqslant K^{-1} \bmod \left(V-J_{n}\right)
$$

so $U-[w, y]$ has definite modulus as well. Similarly, in-radius $\left(V, f^{i}(w)\right) \asymp\left|I_{n}\right| \asymp r$. From the quasiconformality of $f^{-i}$ and the fact that $f \mid S^{1}$ is an isometry, we conclude that in-radius $(U, w) \asymp r$ as well.

The hyperbolic geometry of $\Omega$. By the thick part of $\Omega$ we will mean the region where the injectivity radius of the hyperbolic metric exceeds some small constant $\varepsilon_{0}>0$. Since $\Omega$ is a punctured disk, its thin part is simply a standard horoball neighborhood of the cusp at $z=\infty$. We choose $\varepsilon_{0}$ small enough that $J(f)$ is contained in the thick part.

The hyperbolic metric $\varrho_{\Omega}$ in the thick part is comparable to the $(1 / d)$-metric (cf. [Mc3, Theorem 2.3]); that is,

$$
\varrho_{\Omega}(z)|d z| \asymp \frac{|d z|}{d(z, \partial \Omega)}
$$

The pre-Siegel disk. Let $\iota$ be the involution of the $z$-plane given by $\iota(z)=2 c_{0}-z$. This rotation about the critical point exchanges the two sheets of $f$; that is, $f(\iota(z))=f(z)$.

Let $D^{\prime}=\iota(D)$ be the pre-Siegel disk. Then $f\left(D^{\prime}\right)=D$ and $\bar{D} \cap \overline{D^{\prime}}=\left\{c_{0}\right\}$.

The two quasidisks $D$ and $D^{\prime}$ meet "radially" at $c_{0}$. More precisely, there is a quasiconformal homeomorphism of the plane sending $D \cup D^{\prime}$ to the region bounded by the lemniscate $L=\left\{z:\left|z^{2}-1\right|=1\right\}$ (see Figure 6). Indeed, the critical value of $f$ lies in the quasicircle $\partial D$, so $f^{-1}(\partial D)=\partial\left(D \cup D^{\prime}\right)$ is quasiconformally homeomorphic to the square root of a circle passing through the origin, which is $L$. Thus with respect to the 


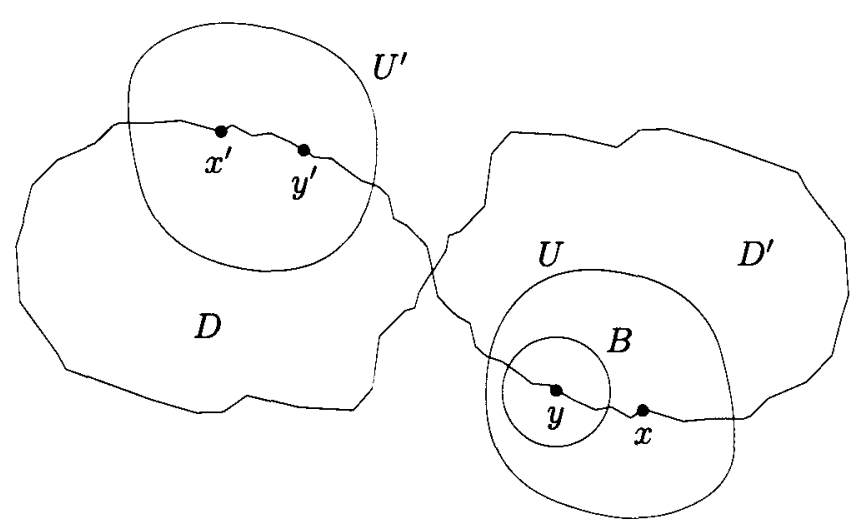

Fig. 7. Univalent maps near points in the boundary of the pre-Siegel disk $D^{\prime}$

hyperbolic metric on $\Omega$, the disk $D^{\prime}$ is contained in a bounded neighborhood of a geodesic ray terminating at the critical point $c_{0}$.

With this background in mind we can now prove

Proposition 3.5. For any $x$ in the thick part of $\Omega$, there is an embedded hyperbolic ball $B$ centered at $y$, and an $i \geqslant 0$, such that

$$
f^{i}:(B, y) \rightarrow\left(B^{\prime}, c_{0}\right)
$$

is univalent, $\operatorname{diam}_{\Omega} B \asymp 1$ and $d_{\Omega}(x, y)=O(1)$.

Proof. In brief, $B$ is constructed as follows. Apply the dynamics to rotate $x$ until it is close to the pre-Siegel disk; then find a nearby point in $\partial D^{\prime}$. After one iterate, the nearby point maps to $\partial D$, and after a suitable further iterate it rotates into a ball $B^{\prime}$ containing the critical point. The preimage of $B^{\prime}$ near $x$ is the desired ball $B$.

For the detailed proof, we first suppose that $x \in \partial D^{\prime}$. Let $x^{\prime}=\iota(x) \in \partial D=P(f)$. For any $r>0$, Theorem 3.4 (and the remarks following) provide a univalent map $f^{i}$ : $\left(U^{\prime}, y^{\prime}\right) \rightarrow\left(V, c_{0}\right)$ such that $d_{U^{\prime}}\left(x^{\prime}, y^{\prime}\right)=O(1)$ and in-radius $\left(U^{\prime}, y^{\prime}\right) \asymp \operatorname{out}-\operatorname{radius}\left(U^{\prime}, y^{\prime}\right) \asymp r$. With suitable $r$ we obtain in-radius $\left(U^{\prime}, y^{\prime}\right) \asymp d\left(y^{\prime}, D^{\prime}\right)$ and out-radius $\left(U^{\prime}, y^{\prime}\right)<d\left(y^{\prime}, D^{\prime}\right)$. In particular, $U^{\prime}$ and $D^{\prime}$ are disjoint.

Now let $(U, y)=\left(\iota\left(U^{\prime}\right), \iota\left(y^{\prime}\right)\right)$. Then $(U, y)$ also maps to $\left(V, c_{0}\right)$ under $f^{i}$, and $U \cap D=\varnothing$, so $U \subset \Omega$. Since $\iota$ is an isometry,

$$
\operatorname{in} \text {-radius }(U, y) \asymp d(y, D)=d(y, \partial \Omega) .
$$

But the hyperbolic metric on $\Omega$ is comparable to $|d z| / d(z, \partial \Omega)$, so there is a hyperbolic ball $B$ of definite radius centered at $y$ and contained in $U$ (see Figure 7); by the Schwarz lemma,

$$
d_{\Omega}(x, y) \leqslant d_{U}(x, y)=d_{U^{\prime}}\left(x^{\prime}, y^{\prime}\right)=O(1)
$$



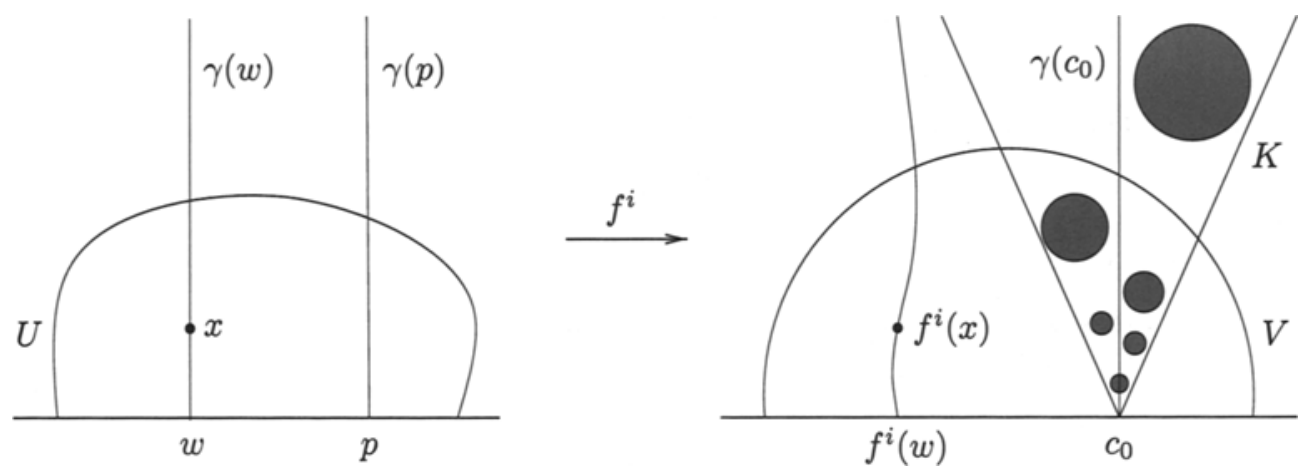

Fig. 8. Pulling back from the cone at the critical point

completing the proof of the proposition for $x \in \partial D^{\prime}$.

The general case of an arbitrary $x$ in the thick part of $\Omega$ is conveniently visualized in the linear coordinate.

For $w \in S^{1}$, let $\gamma(w)=\{r w: 1<r<\infty\} \subset \mathbf{C}_{w}$ denote the ray joining $w$ to infinity. With respect to the hyperbolic metric on $\Omega=\{w:|w|>1\}$, this ray is a geodesic. Since $D^{\prime}$ tends to $c_{0}$ radially, there is a $C$ such that

$$
D^{\prime} \subset K=\left\{w \in \Omega: d_{\Omega}\left(w, \gamma\left(c_{0}\right)\right)<C\right\}
$$

By the first part of the proof, we may also choose $C$ so that the cone $K$ is uniformly filled with hyperbolic balls $(B, y)$ of definite radius mapping univalently over the critical point. See Figure 8.

Given $x$ in the thick part of $\Omega$, choose $w \in S^{1}=\partial D$ so that $x \in \gamma(w)$. By Theorem 3.4 there is a $p \in S^{1}$ and an approximate rotation

$$
f^{i}:(U, p) \rightarrow\left(V, c_{0}\right)
$$

such that $2|x-w|<$ in-radius $(U, w)$ and $|w-p|=O(|w-x|)$. In particular, $d_{\Omega}(x, \gamma(p))=$ $O(1)$. But $F^{i}(\gamma(p))=\gamma\left(c_{0}\right)$, and since $f^{i} \mid U$ is an approximate isometry, we also have $d_{\Omega}\left(f^{i}(x), \gamma\left(c_{0}\right)\right)=O(1)$. Thus $f^{i}(x)$ is near the cone $K$, so there is a hyperbolic ball $\left(B_{0}, y_{0}\right) \subset K \cap V$ of definite radius with $d_{\Omega}\left(x, B_{0}\right)=O(1)$, such that $f^{j}:\left(B_{0}, y_{0}\right) \rightarrow\left(B_{0}^{\prime}, c_{0}\right)$ is univalent for some $j>0$. Then $f^{-i}\left(B_{0}\right)$ contains a hyperbolic ball $B$ of definite radius centered at $y=f^{-i}\left(y_{0}\right)$, we have $d_{\Omega}(x, B)=O(1)$, and

$$
f^{i+j}:(B, y) \rightarrow\left(B^{\prime}, c_{0}\right)
$$

is the desired univalent mapping. 
Expansion in the Julia set. To establish Theorem 3.2, we need to find disks mapping univalently over the critical point at every scale near every point in the Julia set. To populate small scales, we will use expansion relative to the hyperbolic metric on $\Omega$.

Quite generally, for any polynomial map $f: \mathbf{C} \rightarrow \mathbf{C}$ with $|P(f)|>1$, we have $\left\|f^{\prime}(x)\right\| \geqslant 1$ with respect to the hyperbolic metric on $\mathbf{C}-P(f)$ (so long as $f(x) \notin P(f)$ ). Moreover, if $x \in J(f)$, then either the forward orbit of $x$ lands in $P(f)$ or $\left\|f^{i}(x)\right\| \rightarrow \infty$. These statements follow from the Schwarz lemma, the forward invariance of $P(f)$ and the fact that $J(f) \subset \overline{\bigcup f^{-i}(P(f))}$. See $[\mathrm{Mc3}, \S 3.2]$.

In the case at hand, $\mathbf{C}-P(f)=\Omega \cup D$. Of course $f \mid D$ is an isometry, but $f$ expands the hyperbolic metric on $\Omega$. Note too that $J(f) \subset P(f) \cup \Omega$, so the orbit of a point in the Julia set can exit $\Omega$ only by landing in the boundary of the Siegel disk.

For a vector $v$ in the tangent space to a point $z \in \mathbf{C}$, we let $|v|$ and $l(v)$ denote the lengths of $v$ in the Euclidean metric and in the hyperbolic metric on $\mathbf{C}-P(f)$ respectively. We set $l(v)=\infty$ if $z \in P(f)$.

We may now complete the

Proof of Theorem 3.2 (Nearby critical visits). Let $z_{0}=z \in J(f)$ and $r>0$ be a given point in the Julia set and a given scale. Let $v_{0} \in T_{z} \mathbf{C}$ be a vector with $\left|v_{0}\right|=r$, let $z_{i}=f^{i}\left(z_{0}\right)$ and let $v_{i} \in T_{z_{i}} \mathbf{C}$ be the image of $v_{0}$ under $\left(f^{i}\right)^{\prime}$. Then

$$
l\left(v_{0}\right) \leqslant l\left(v_{1}\right) \leqslant l\left(v_{2}\right) \leqslant \ldots \quad \text { and } \quad l\left(v_{i}\right) \rightarrow \infty
$$

We wish to find a univalent map $f^{i}:(U, y) \rightarrow\left(V, c_{0}\right)$ with in-radius $(U, y) \asymp r$ and $|y-z|=O(r)$. To do so, we distinguish three cases.

Case I. $l\left(v_{0}\right) \geqslant 1$. (We include the case $l\left(v_{0}\right)=\infty$, which arises when $z_{0} \in P(f)$.) In this case, there is a point $z^{\prime} \in P(f)$ with $\left|z-z^{\prime}\right|=O(r)$, since the hyperbolic metric on $\Omega$ at $z$ is comparable to the $(1 / d)$-metric. Applying Theorem 3.4, we obtain a univalent map $f^{i}:(U, y) \rightarrow\left(V, c_{0}\right)$ with in-radius $(U, y) \asymp r$ and $\left|y-z^{\prime}\right|=O(r)$. Then $\mid z-y_{\mid}=O(r)$ and we are done.

Case II. There exists a $j$ such that $l\left(v_{j-1}\right) \leqslant \varepsilon$ but $l\left(v_{j}\right) \geqslant 1$. Here $\varepsilon>0$ depends only on $\theta$; its value is chosen below.

By Case I there is a univalent map $f^{i}:\left(U^{\prime}, y^{\prime}\right) \rightarrow\left(V, c_{0}\right)$ with in-radius $\left(U^{\prime}, y^{\prime}\right) \asymp\left|v_{j}\right|$ and $\left|y^{\prime}-z_{j}\right|=O\left(\left|v_{j}\right|\right)$. Replacing $U^{\prime}$ with a ball centered at $y$, we may also assume that $\operatorname{diam}\left(U^{\prime}\right) \asymp\left|v_{j}\right|$.

We claim that $\left(U^{\prime}, y^{\prime}\right)$ can be pulled back by $f^{j}$ to yield the desired disk $(U, y)$ for $z_{0}$. To see this, first note that the condition

$$
\varepsilon>l\left(v_{j-1}\right) \asymp \frac{\left|v_{j-1}\right|}{d\left(z_{j-1}, P(f)\right)}
$$


implies that there is a ball $B_{j-1}=B\left(z_{j-1}, s\right)$, disjoint from the postcritical set $P(f)$, with $s \asymp\left|v_{j-1}\right| / \varepsilon$. Then $f \mid B_{j-1}$ is univalent. By the Koebe $\frac{1}{4}$-theorem, $B_{j}=f\left(B_{j-1}\right)$ contains a ball of radius comparable to $\left|v_{j}\right| / \varepsilon$ centered at $z_{j}$. Thus we may choose $\varepsilon$ sufficiently small (depending only on $\theta$ ) such that $U^{\prime}$ is contained well within $B_{j}$.

Let $f^{-j}: B_{j} \rightarrow B_{0}$ be the univalent inverse branch of $f^{j}$ sending $z_{j}$ to $z_{0}$. This map exists because $B_{j-1}$ is disjoint from $P(f)$, and because $f: B_{j-1} \rightarrow B_{j}$ is univalent. Let $(U, y)=f^{-j}\left(U^{\prime}, y^{\prime}\right)$. By the Koebe distortion theorem, $f^{-j}$ is approximately a similarity with factor $\left|v_{0}\right| /\left|v_{j}\right|$ near $z_{j}$. Since $U^{\prime}$ is contained well within $B_{j}$, we have in-radius $(U, y) \asymp\left|v_{0}\right|,\left|z_{0}-y\right|=O\left(\left|v_{0}\right|\right)$, and $f^{i+j}:(U, y) \rightarrow\left(V, c_{0}\right)$ is univalent. This completes the proof of Case II.

Case III. There exists a $j$ such that $\varepsilon<l\left(v_{j}\right)<1$. By Proposition 3.5, there is a centered hyperbolic ball $\left(B, y^{\prime}\right) \subset \Omega$ with $\operatorname{diam}_{\Omega}(B) \asymp 1$ and $d_{\Omega}\left(z_{j}, y^{\prime}\right)=O(1)$, such that $f^{i}:\left(B, y^{\prime}\right) \rightarrow\left(B^{\prime}, c_{0}\right)$ is univalent. Let $\gamma$ be a minimal-length geodesic segment joining $z_{j}$ to $B$. Then $\gamma \cup B$ is simply-connected and disjoint from $P(f)$, so it supports a unique branch of $f^{-j}$ sending $z_{j}$ back to $z_{0}$.

Let $(U, y)=f^{-j}\left(B, y^{\prime}\right)$; then $f^{i+j}:(U, y) \rightarrow\left(V, c_{0}\right)$ is univalent. To complete the proof of this case, we just need to check that in-radius $(U, y) \asymp\left|v_{0}\right|$ and $|z-y|=O\left(\left|v_{0}\right|\right)$. Equivalently, in the hyperbolic metric on $\Omega$, it suffices to check that $d_{\Omega}(z, y)=O\left(l\left(v_{0}\right)\right)$ and $\operatorname{in}_{\operatorname{radius}}(U, y) \asymp l\left(v_{0}\right)$.

Now $\left(f^{-j}\right)^{\prime}\left(z_{j}\right)$ sends the vector $v_{j}$ with $l\left(v_{j}\right) \asymp 1$ to the vector $v_{0}$. Since we have $\operatorname{diam}_{\Omega}(\gamma \cup B)=O(1)$, and the Julia set is contained in the thick part of $\Omega$, the map $f^{-j}$ is approximately a contraction by the constant factor $l\left(v_{0}\right)$ on $\gamma \cup B$ (cf. [Mc3, Theorem 3.8]). But the bounds provided for $(B, y)$ by Proposition 3.5 then imply

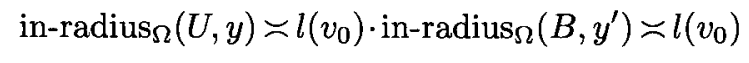

and

$$
d_{\Omega}(z, y) \asymp l\left(v_{0}\right) \cdot d_{\Omega}\left(z_{j}, y^{\prime}\right)=O\left(l\left(v_{0}\right)\right)
$$

as desired.

End of the proof. Since $l\left(v_{j}\right) \rightarrow \infty$, one of the three cases above must hold, and the proof of the theorem is complete.

The argument above follows the proof that there are "small Julia sets everywhere" for certain infinitely renormalizable quadratic polynomials, given in [Mc4, §8.3]. 


\section{Geometry of the Julia set}

In this section we prove that the dimension of the Julia set is less than two, that the critical point is a deep point of $K(f)$, and that the full dynamics of $f$ is uniformly twisting on $J(f)$. A more complete development of the ideas of shallow and deep points, geometric limits of dynamical systems and uniform twisting appears in [Mc4].

Dimension. The Hausdorff dimension of a compact metric space $X$, denoted $H \cdot \operatorname{dim}(X)$, is the infimum of those $\delta>0$ such that $X$ can be covered by balls $B\left(x_{i}, r_{i}\right)$ with $\sum r_{i}^{\delta}$ arbitrarily small. The (upper) box dimension is given by

$$
\text { box-dim }(X)=\limsup _{r \rightarrow 0} \frac{\log N(X, r)}{\log (1 / r)}
$$

where $N(X, r)$ is the minimum number of $r$-balls required to cover $X$. Let us identify the Riemann sphere $\widehat{\mathbf{C}}$ with the sphere at infinity $S_{\infty}^{2}$ for hyperbolic space $\mathbf{H}^{3}$. A closed set $\Lambda \subset S_{\infty}^{2}$ is shallow if every point in its hyperbolic convex hull $K \subset \mathbf{H}^{3}$ is within a uniformly bounded distance of $\partial K$. (Such sets are also called porous.)

A compact set $\Lambda \subset \mathbf{C}$ is shallow if and only if for any $z \in \Lambda$ and $r<1$, there is a ball $B$ disjoint from $\Lambda$ with $\operatorname{diam} B \asymp r$ and $d(z, B)=O(r)$. For example, any quasicircle is shallow. The inequality $\operatorname{H} \cdot \operatorname{dim}(X) \leqslant$ box-dim $(X)$ holds for any metric space, and it is easy to see that box-dim( $\Lambda)<2$ if $\Lambda \subset S_{\infty}^{2}$ is shallow.

THEOREM 4.1 (Dimension of $J$ ). The Julia set $J(f)$ is shallow, and thus

$$
\operatorname{H.dim}(J(f)) \leqslant \operatorname{box}-\operatorname{dim}(J(f))<2 \text {. }
$$

Proof. Since the Siegel disk $D$ is a quasidisk, it fills definite space at every scale around the critical point $c_{0} \in \partial D$. More precisely, for any $s<1$ there is a ball $B \subset$ $B\left(c_{0}, s\right) \cap D$ with $\operatorname{diam} B \asymp s$.

Now consider a point $z \in J(f)$ and a scale $r<1$. By Theorem 3.2, there is a univalent map

$$
f^{i}:(U, y) \rightarrow\left(V, c_{0}\right)
$$

with $|y-z|=O(r)$ and in-radius $(U, y) \asymp r$. Let $s=$ in-radius $\left(c_{0}, V\right)$. Choose a ball $B \subset$ $B\left(c_{0}, s\right) \cap D$ with $\operatorname{diam} B \asymp s$. By the Koebe distortion theorem, $f^{-i}(B)$ contains a ball $B^{\prime}$ with $\operatorname{diam} B^{\prime} \asymp r$ and $d\left(y, B^{\prime}\right)=O(r)$. Since $f^{i}\left(B^{\prime}\right) \subset D$, this ball is disjoint from the Julia set, and since $d\left(z, B^{\prime}\right) \leqslant d(z, y)+d\left(y, B^{\prime}\right)=O(r)$, we have shown that the Julia set is shallow.

As remarked above, the dimension bounds follow. 
Deep points. Let $\Lambda \subset S_{\infty}^{2}$ be a compact set with convex hull $K \subset \mathbf{H}^{3}$. A point $x \in$ $\Lambda \subset S_{\infty}^{2}$ is a deep point of $\Lambda$ if there is a geodesic ray $\gamma:[0, \infty) \rightarrow K$, converging to $x$ and parameterized by hyperbolic arc length, such that

$$
d(\gamma(s), \partial K) \geqslant \varepsilon s>0
$$

for all $s>0$.

One can easily check that $x \in \Lambda \subset \mathbf{C}$ is deep if and only if there is a $\delta>0$ such that for all $r<1$,

$$
B(y, s) \subset B(x, r)-\Lambda \quad \Rightarrow \quad s=O\left(r^{1+\delta}\right) .
$$

For example, a shallow set has no deep points.

By the classification of stable regions, every point in the interior of $K(f)$ lands in the Siegel disk $D$ under forward iteration. Thus $D$ acts like an attractor. Let

$$
K_{\varepsilon}(f)=\left\{z \in \mathbf{C}: d\left(f^{n}(z), D\right) \leqslant \varepsilon \text { for all } n \geqslant 0\right\} .
$$

Clearly $K_{\varepsilon}(f) \subset K(f)$; it consists of points whose orbits stay near the Siegel disk.

THeorem 4.2 (Deep points). The critical point $c_{0}$ is a deep point of $K(f)$. More generally, $z$ is a deep point of $K_{\varepsilon}(f)$ for any $z \in P(f)$ and any $\varepsilon>0$.

A point $x \in \Lambda \subset \mathbf{C}$ is a measurable deep point if for some $\delta>0$ we have

$$
\operatorname{area}(B(x, r)-\Lambda)=O\left(r^{2+\delta}\right)
$$

for all $r>0$. This condition means that the density of $\Lambda$ in $B(x, r)$ tends to 1 exponentially fast (it is bounded below by $1-C r \delta$ ).

The next result also appears in [Mc4, Proposition 2.24].

Proposition 4.3. If $x$ is a deep point of $\Lambda$ and $\partial \Lambda$ is shallow, then $x$ is a measurable deep point of $\Lambda$.

Proof. Consider a square $S$ of size $r$ centered at $x$. Since $x$ is a deep point, there is an $\alpha>0$ such that every point of $X=S-\Lambda$ lies within distance $r^{1+\alpha}$ of $\partial \Lambda$. On the other hand, since $\partial \Lambda$ is shallow, there is a $\delta<2$ such that if we cut $S$ into subsquares of size $\asymp r^{1+\alpha}$, whose total number is $O\left(r^{-2 \alpha}\right)$, it only takes $O\left(r^{-\delta \alpha}\right)$ of them to cover $X \cap S$. Thus the area of $X \cap S$ is $O\left(r^{2+(2-\delta) \alpha}\right)$, and $x$ is a measurable deep point.

Since $J(f)=\partial K(f)$ is shallow, we may deduce

Corollary 4.4. A random $z$ near the critical point has a high probability of lying in $K(f)$. More precisely, there is a $\delta>0$ such that for small $r$, we have

$$
\operatorname{area}\left(B\left(c_{0}, r\right)-K(f)\right)=O\left(r^{2+\delta}\right) .
$$

More generally one sees 
Corollary 4.5. Every $z \in P(f)$ is a measurable deep point of $K_{\varepsilon}(f)$.

Proof. Choose a smoothly bounded disk $A$ with $\bar{D}$ in its interior, lying within an $\varepsilon$-neighborhood of $D$ and outside the part of $\mathbf{C}-J(f)$ where the hyperbolic injectivity radius is less than one. Let $K_{A}(f)=\bigcap f^{-n}(A)$. Then $K_{\delta}(f) \subset K_{A}(f) \subset K_{\varepsilon}(f)$ for some $\delta>0$. Since $z$ is a deep point of $K_{\delta}(f)$, to prove the corollary it suffices to show that $\partial K_{A}(f)$ is shallow.

Consider a point $w \in \partial K_{A}(f)$ and a scale $r<1$. We must find a ball disjoint from $\partial K_{A}(f)$, within distance $r$ of $w$ and with radius $\asymp r$.

By shallowness of the Julia set, there is such a ball $B\left(z_{1}, r_{1}\right)$ disjoint from $J(f)$. If $B\left(z_{1}, \frac{1}{2} r_{1}\right)$ is disjoint from $\partial K_{A}(f)$ we are done; otherwise, there is a $B\left(z_{2}, \frac{1}{2} r_{1}\right) \subset$ $B\left(z_{1}, r_{1}\right)$ with $z_{2} \in \partial K_{A}(f)-J(f)$. Then $f^{n}\left(z_{2}\right) \in \partial A$ for some $n$. The ball $U=B\left(z_{2}, \frac{1}{10} r_{1}\right)$ has diameter less than one in the hyperbolic metric on $\mathbf{C}-J(f)$, so $f^{n} \mid U$ is injective. Since $\partial A$ is smooth, $f^{n}(U)-A$ contains a ball $B$ with $\operatorname{diam} B \asymp \operatorname{diam} f^{n}(U)$, and by Koebe $f^{-n}(B) \subset U$ contains a ball $B\left(z_{3}, r_{3}\right)$ disjoint from $K_{A}(f)$ with $r_{3} \asymp r$.

Escape from the Siegel disk. To prove that $c_{0}$ is a deep point of $K(f)$, we will analyze the orbit of a point $z$ that starts near $c_{0}$ but escapes to infinity. While the orbit is near $D$, it shadows a rotating orbit until it comes close to the critical point. As it passes the critical point, the orbit can move much closer to $D$, but it can only move farther away by a bounded factor. Thus before $f^{i}(z)$ can escape from the influence of $D$, it must make many visits to the critical point. It turns out that each visit results in definite expansion with respect to the hyperbolic metric on $\Omega=\mathbf{C}-\bar{D}$. On the last visit, there is a point $y^{\prime}$ in $\partial D^{\prime}=\iota(\partial D)$ at a bounded hyperbolic distance from $f^{i}(z)$. Pulling this point back by $f^{-i}$, we obtain a point $y \in K(f)$ very close to $z$. This point limits the radius of a ball $B(z, s) \subset B\left(c_{0}, r\right)-K(f)$, and shows that $c_{0}$ is deep.

To measure the rate of escape from the influence of Siegel disk $D$, we will work in the linear coordinate $w$. Let $L(w)=\log d(w, D)$; that is,

$$
L(w)= \begin{cases}\log (|w|-1), & |w|>1 \\ -\infty & \text { otherwise }\end{cases}
$$

If $w \in U, w$ is near $D$ and $f^{i} \mid U$ is an approximate rotation (in the sense of $\S 3$ ), then

$$
L\left(f^{i}(w)\right)=L(w)+O(1)
$$

while for all $w$ near $D$ we still have the weaker statement

$$
L(f(w)) \leqslant L(w)+O(1)
$$


To see (4.1), first note that near the critical point (recalling that $c_{1}$ is the critical value),

$$
\left|f(w)-c_{1}\right| \asymp\left|w-c_{0}\right|,
$$

because $f$ is quasiregular and $f \mid S^{1}$ is an isometry. (The branching behavior of $f$ is similar to that of the map $(r, \theta) \mapsto(r, 2 \theta)$ in polar coordinates.) If $d(w, D) \ll d\left(w, c_{0}\right)$, then $f$ is an approximate rotation at $w$, so $L(f(w))=L(w)+O(1)$; otherwise $d(f(w), D) \leqslant$ $d\left(f(w), c_{1}\right) \asymp d\left(w, c_{0}\right) \asymp d(w, D)$, which gives $(4.1)$.

Proof of Theorem 4.2 (Deep points). We will first prove that every point of $P(f)$ is a deep point of $K(f)$, deferring the case of $K_{\varepsilon}(f)$ to the end.

We will actually show that there is an $\alpha>0$ so that for all $z$ in the thick part of $\Omega$, we have

$$
d_{\Omega}(z, K(f))=O\left(d(z, D)^{\alpha}\right) .
$$

Since the Poincaré metric on $\Omega$ is comparable to the $(1 / d)$-metric, this bound implies $d(z, K(f))=O\left(d(z, P(f))^{1+\alpha}\right)$, from which it follows easily that every point in the postcritical set is (uniformly) deep in $K(f)$.

Let us also remark that

$$
f:\left(\mathbf{C}-\overline{D \cap D^{\prime}}\right) \rightarrow(\mathbf{C}-\bar{D})=\Omega
$$

is a covering map, and hence an isometry between the respective hyperbolic metrics. The inclusion between domain and range is a contraction, by the Schwarz lemma. The amount of contraction is bounded in terms of $d_{\Omega}\left(z, D^{\prime}\right)$. Thus the expansion of $f$, measured by $\left\|f^{\prime}(z)\right\|$ using the hyperbolic metric of $\Omega$ at both $z$ and $f(z)$, satisfies

$$
\left\|f^{\prime}(z)\right\|>C(d)>1
$$

where $d=d_{\Omega}\left(z, D^{\prime}\right)$. Compare [Mc4, Proposition 4.4.2].

We now turn to the proof of (4.2). Let $z_{0}=z$, and $z_{j}=f^{j}\left(z_{0}\right)$ for $j>0$. Applying Theorem 3.4 to the point $p$ in $P(f)$ nearest $z_{0}$, we obtain an approximate rotation $f^{i_{1}}: U \rightarrow V$ such that $z_{0} \in U$ and $d_{\Omega}\left(z_{i_{1}}, \partial D^{\prime}\right)=O(1)$. (Here we use the fact that $D^{\prime}$ converges radially to $c_{0}$.) Then $\left\|f^{\prime}\left(z_{i_{1}}\right)\right\|>\eta>1$, where $\eta$ depends only on the rotation number $\theta$. On the other hand, $\left|L\left(z_{j}\right)-L\left(z_{0}\right)\right|=O(1)$ for $0 \leqslant j \leqslant i_{1}$, and by (4.1) we have

$$
L\left(z_{i_{1}+1}\right) \leqslant L\left(z_{0}\right)+O(1)
$$

Now repeat the argument starting with $z_{i_{1}+1}$, to obtain a second visit $z_{i_{2}}$ within a bounded distance of $D^{\prime}$. Continuing in this way, we construct a sequence of visits $z_{i_{k}}$ 
along which $L\left(z_{i_{k}}\right)$ increases at most linearly in $k$. The construction terminates when the orbit gets a definite distance from $D$, say when $L\left(z_{i_{k}}\right)>0$. Because of the linear increase of $L\left(z_{i_{k}}\right)$, the sequence is defined at least for $k=1,2, \ldots, N$ where $N \asymp-L\left(z_{0}\right)$. But $L\left(z_{0}\right) \asymp \log d\left(z_{0}, D\right)$ (by Hölder continuity of the quasiconformal map $\Phi: \mathbf{C}_{z} \rightarrow \mathbf{C}_{w}$ ), and thus

$$
N \asymp \log \left(\frac{1}{d\left(z_{0}, D\right)}\right) .
$$

Since there are $N-1$ visits past $D^{\prime}$, we have $\left\|f^{i_{N}}\left(z_{0}\right)\right\| \geqslant \eta^{N-1}$. Join $z_{i_{N}}$ to a point $y^{\prime} \in \partial D^{\prime}$ by an arc $\gamma^{\prime}$ of hyperbolic length $O(1)$. Then $y^{\prime} \in K(f)$, and since $\gamma^{\prime}$ is disjoint from $P(f)$ it lifts under $f^{-i_{N}}$ to an arc $\gamma$ joining $z_{0}$ to $y \in K(f)$ with hyperbolic length $O\left(\eta^{-N}\right)$. Thus by (4.3),

$$
d_{\Omega}\left(z_{0}, K(f)\right) \leqslant d_{\Omega}\left(z_{0}, y\right)=O\left(\eta^{-N}\right)=O\left(d\left(z_{0}, D\right)^{\alpha}\right)
$$

for some $\alpha>0$ depending only on $\theta$. This completes the proof that each point in the postcritical set is a deep point of $K(f)$.

To handle the case of $K_{\varepsilon}(f)$, we modify the construction so that it terminates when $L\left(z_{i_{k}}\right)>M(\varepsilon)$. Then $L\left(z_{j}\right)<M(\varepsilon)+O(1)$ for $j=0, \ldots, i_{N}$. For suitable choice of $M(\varepsilon)$, this condition insures that $z_{j}$ lies well within an $\varepsilon$-neighborhood of $D$. Now each $y_{j}=f^{j}(y)$, $j=0, \ldots, i_{N}$, is at a bounded hyperbolic distance from $z_{j}$, so it too is no farther than $\varepsilon$ from $D$. Finally $f^{i_{N}}(y)=y^{\prime} \in \partial D^{\prime}$, so $\left.d\left(f^{j}(y), D\right)=0\right)$ for $j>i_{N}$. Thus $y \in K_{\varepsilon}(f)$ and we have shown that every point of $P(f)$ is also a deep point of $K_{\varepsilon}(f)$.

To conclude this section we will prove

THEOREM 4.6 (Uniform twisting). The holomorphic dynamical system $(\mathcal{F}(f), J(f))$ is uniformly twisting.

This theorem illustrates one of the parallels summarized in Table 2. Although the result is not used in the sequel, we include it because the same result holds for any infinitely renormalizable real quadratic map $f(z)=z^{2}+c$ with bounded combinatorics $[\mathrm{Mc4}, \S 9.4]$.

A dynamical system $(\mathcal{F}, \Lambda)$ is uniformly twisting if there is robust nonlinearity at every scale about every point in $\Lambda$. This concept is naturally formulated in terms of geometric limits; a complete development appears in $[\mathrm{Mc} 4, \S 9]$. For the purpose of the present discussion, we will simply state a sufficient criterion for uniform twisting.

Let $\mathcal{H}$ denote the space of all holomorphic maps $g: U \rightarrow \widehat{\mathbf{C}}$ such that $U$ is an open set of $\widehat{\mathbf{C}}$. We give $\mathcal{H}$ the following (non-Hausdorff) topology: $g_{n}: U_{n} \rightarrow \widehat{\mathbf{C}}$ tends to $g: U \rightarrow \widehat{\mathbf{C}}$ if for every compact $K \subset U$, we have $K \subset U_{n}$ for all $n$ sufficiently large and $g_{n} \rightarrow g$ uniformly on $K$. 
Given a rational map $f: \widehat{\mathbf{C}} \rightarrow \widehat{\mathbf{C}}$, let

$$
\mathcal{F}(f)=\left\{g: U \rightarrow \widehat{\mathbf{C}}: \text { for some } i, j \geqslant 0, f^{i} \circ g=f^{j}\right\} \subset \mathcal{H} .
$$

PROPOSITION 4.7. Let $\Lambda \subset \mathbf{C}$ be compact. Suppose that for any sequence

$$
A_{n}(z)=\alpha_{n}\left(z-\beta_{n}\right)
$$

with $\alpha_{n} \rightarrow \infty$ and $\beta_{n} \in \Lambda$, there exist $g_{n} \in \mathcal{F}(f)$ such that after passing to a subsequence,

$$
A_{n} \circ g_{n} \circ A_{n}^{-1} \rightarrow h \in \mathcal{H}
$$

where $h: U \rightarrow V$ is a nonconstant map with a critical point.

Then $(\mathcal{F}(f), \Lambda)$ is uniformly twisting.

This criterion reduces the proof of uniform twisting to

Proposition 4.8. For every $x \in J(f)$ and $r<1$, there is a proper degree-two map $g \in \mathcal{F}(f)$,

$$
g:(U, u) \rightarrow(V, v)
$$

such that $U$ and $V$ are disks, $g^{\prime}(u)=0$, in-radius $(U, u) \asymp$ in-radius $(V, v) \asymp r,|x-u|=O(r)$ and $|x-v|=O(r)$.

Proof. First assume $x=c_{0}$. Then $g$ can be taken to be a first return map near the critical point. To make this precise, we prove the result in the linear coordinate $w$. Choose $n$ such that $\left\|q_{n} \theta\right\| \asymp r$. Let $V$ be the round disk orthogonal to $S^{1}$, meeting the circle in the interior of the arc $\left[q_{n} \theta, q_{n+1} \theta\right]$, and let $i=q_{n}+q_{n+1}$. Then $f^{i}$ has a unique critical value $v \in V$, with angular coordinate $\left(q_{n}+q_{n+1}\right) \theta$. Since $\theta$ has bounded type, in-radius $(V, v) \asymp r$. Let $U$ be the unique component of $f^{-i}(V)$ containing $c_{0}$. Then

$$
g=f^{i}:\left(U, c_{0}\right) \rightarrow(V, v)
$$

is proper of degree two, and it is easy to see that in-radius $\left(U, c_{0}\right) \asymp r$, so the result is established for $x=c_{0}$. Moreover, we can shrink $U$ and $V$ slightly so that their diameters are comparable to $r$.

To treat the case of an arbitrary $z \in J(f)$, we use Theorem 3.2 to find a univalent map $f^{j}:(U, u) \rightarrow\left(V, c_{0}\right)$ at scale $r$ near $z$. Pulling back a map $\tilde{g}=f^{i}$ which works at a scale $s \asymp$ in-radius $\left(V, c_{0}\right)$ near the critical point, we obtain a map $g=f^{-j} \circ f^{i+j}$ which works at scale $r$ near $z$, completing the proof. 
Proof of Theorem 4.6 (Uniform twisting). Given $A_{n}(z)=\alpha_{n}\left(z-\beta_{n}\right)$ as in Proposition 4.7, apply the preceding proposition with $x=\beta_{n}$ and $r=1 / \alpha_{n}$ to obtain a sequence of maps $g_{n} \in \mathcal{F}(f)$ such that

$$
h_{n}=A_{n} \circ g_{n} \circ A_{n}^{-1}:\left(U_{n}, u_{n}\right) \rightarrow\left(V_{n}, v_{n}\right)
$$

is proper of degree two, $h_{n}^{\prime}\left(u_{n}\right)=0$, in-radius $\left(U, u_{n}\right) \asymp$ in-radius $\left(V_{n}, v_{n}\right) \asymp 1,\left|u_{n}\right|=O(1)$ and $\left|v_{n}\right|=O(1)$. In the Carathéodory topology, a subsequence $h_{n_{k}}$ converges to a degree-two proper map $h:(U, u) \rightarrow(V, v)$ with $h^{\prime}(u)=0[\mathrm{Mc} 3$, Theorem 5.6], and the same subsequence tends to $h$ in $\mathcal{H}$.

\section{Universality}

A polynomial-like map $g: U \rightarrow V$ is a proper holomorphic map between disks in $\mathbf{C}$, such that $\bar{U}$ is a compact subset of $V$. A quadratic-like map is a polynomial-like map of degree two.

The filled Julia set $K(g)$ is the set of points which never escape under iteration; it is defined by

$$
K(g)=\bigcap_{1}^{\infty} g^{-i}(V)
$$

Similarly, the postcritical set $P(g)$ is the closure of the forward orbit of the critical points of $g$.

Let $g_{i}: U_{i} \rightarrow V_{i}, i=1,2$, be polynomial-like maps of the same degree. A hybrid conjugacy is a quasiconformal map $\phi$ between neighborhoods of $K\left(g_{1}\right)$ and $K\left(g_{2}\right)$, conjugating $g_{1}$ to $g_{2}$, with $\bar{\partial} \phi=0$ a.e. on $K\left(g_{1}\right)$. We say that $g_{1}$ and $g_{2}$ are hybrid equivalent if such a conjugacy exists.

A fundamental result of Douady and Hubbard states that every polynomial-like map $g$ is hybrid equivalent to a polynomial of the same degree. If $K(g)$ is connected then $f$ is unique up to conformal conjugacy [DH1, Theorem 1].

THEOREM 5.1 (Universality). Let $\theta$ be an irrational of bounded type, and let $g$ and $h$ be quadratic-like maps with fixed points of multiplier $\exp (2 \pi i \theta)$. Then:

(1) there is a hybrid conjugacy $\phi$ between $g$ and $h$;

(2) the complex derivative $\phi^{\prime}(z)$ exists for all $z \in P(g)$; and

(3) there are $M, \alpha, \delta>0$ so that for all $z \in P(g)$ and all $t$ with $|t|<\delta$, we have

$$
\left|\frac{\phi(z+t)-\phi(z)}{t}-\phi^{\prime}(z)\right| \leqslant M|t|^{\alpha} .
$$

By the Whitney extension theorem [St, §VI.2.3] we have: 
Corollary 5.2. The derivative $\phi^{\prime}(z)$ is Hölder continuous on $P(g)$, and $\phi \mid P(g)$ extends to a continuous, $C^{1+\alpha}$-function on the whole plane.

CoRollary 5.3. The Hausdorff dimension of $P(g)$ is equal to that of $P(f)$, where $f(z)=e^{2 \pi i \theta} z+z^{2}$.

Proof. By (5.1) a hybrid conjugacy from $f$ to $g$ is bi-Lipschitz on the postcritical set, and such maps preserve dimension.

We now proceed to the proof of Theorem 5.1.

The quadratic-like maps $g$ and $h$ are each hybrid equivalent to a quadratic polynomial. It is not hard to see that a hybrid conjugacy preserves the multiplier of any indifferent cycle; thus $g$ and $h$ are hybrid equivalent to $f(z)=e^{2 \pi i \theta} z+z^{2}$, and hence to each other.

For convenience we let $\phi: \mathbf{C} \rightarrow \mathbf{C}$ denote a quasiconformal map of the whole plane which restricts to a hybrid conjugacy from $g$ to $h$. Let us say that $\phi$ is $C^{1+\alpha}$-conformal at $z$ if $\phi^{\prime}(z)$ exists and

$$
\phi(z+t)=\phi(z)+\phi^{\prime}(z) \cdot t+O\left(|t|^{1+\alpha}\right) .
$$

Thus (5.1) says that $\phi$ is uniformly $C^{1+\alpha}$-conformal on $P(f)$.

The following result appears in $[\mathrm{Mc} 4, \S 2.6]$ :

ThEOREM 5.4 (Boundary conformality). Let $\phi: \mathbf{C} \rightarrow \mathbf{C}$ be a quasiconformal map with $\bar{\partial} \phi=0$ on a measurable set $\Omega$, and let $x$ be a measurable deep point of $\Omega$. Then $\phi$ is $C^{1+\alpha}$-conformal at $x$.

COROLlaRY 5.5. The hybrid conjugacy $\phi$ is $C^{1+\alpha}$ at the critical point $c_{0}(g)$.

Proof. By Corollary 4.4, the critical point $c_{0}(f)$ is a measurable deep point of the interior of $K(f)$ (since $J(f)$ has measure zero). This deepness property is preserved by quasiconformal maps (using e.g. the estimates of [As], so $c_{0}(g)$ is a measurable deep point of $\Omega=\operatorname{int} K(g)$. But $\phi$ is conformal on $\Omega$, so $\phi$ is $C^{1+\alpha}$ at $c_{0}(g)$.

One way to now complete the proof of Theorem 5.1 is to observe that all points in $P(f)$ are measurable deep points of $K(f)$, with uniform bounds, so $\phi$ is uniformly $C^{1+\alpha}$ on $P(f)$, by an effective version of Theorem 5.4. We will proceed differently, however, to illustrate directly that smoothness along the post-critical set is inherited from smoothness at the critical point.

Distortion of triangles. Let

$$
D_{t}(z)=\frac{\phi(z+t)-\phi(z)}{t}
$$




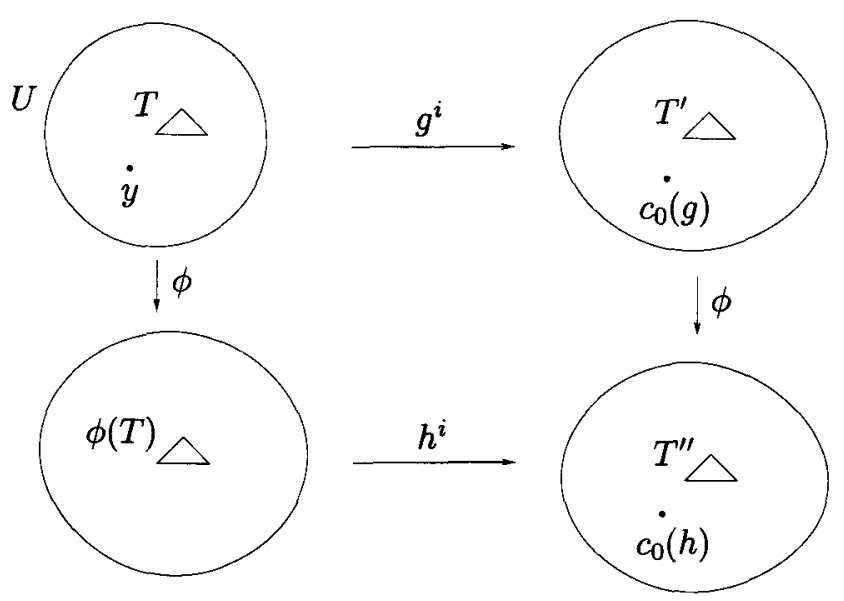

Fig. 9. Nearly similar triangles

and define

$$
N_{t}(z)=\sup _{1 / 2<|t / s|<2}\left|\frac{D_{t}(z)}{D_{s}(s)}-1\right| .
$$

By a calculus argument (controlling $\log D_{t}(z)$ with a geometric series) one has

LEMMA 5.6. Suppose that $N_{t}(z)<C|t|^{\alpha}$ for all $z$ in a compact set $K$ and all $t$ sufficiently small. Then $\phi$ is uniformly $C^{1+\alpha}$ on $K$.

Now think of an ordered triple of points $T=(a, b, c) \subset \mathbf{C}$ as a triangle, with shape determined (up to similarity) by the ratio $\sigma(T)=(b-a) /(c-a)$. Then

$$
N_{t}(z) \leqslant 2 \sup _{T}|\sigma(\phi(T))-\sigma(T)|
$$

where the sup is over all triangles $T=(z, z+t, z+s)$ with $|\sigma(T)|$ in $\left[\frac{1}{2}, 2\right]$. So to show that $\phi$ is uniformly $C^{1+\alpha}$ on $P(f)$, we need only check that

$$
|\sigma(\phi(T))-\sigma(T)|=O\left(|t|^{\alpha}\right)
$$

when $z \in P(f)$ and $t$ is sufficiently small.

To obtain this estimate, we will use the dynamics to move $T$ and $\phi(T)$ close to the critical point. By the Koebe distortion theorem, the shapes of these triangles are almost preserved if the dynamics is univalent on a large neighborhood. On the other hand, $\phi$ is smooth at the critical point, so the shape is nearly preserved there as well. See Figure 9.

Completion of the proof of Theorem 5.1 (Universality). By Corollary 5.5, there is a $\beta>0$ such that $\phi$ is $C^{1+\beta}$-conformal at the critical point $c_{0}(g)$. 
Consider a small $\varepsilon>0$, whose value is to be chosen shortly. Since $g$ is hybrid equivalent to a quadratic polynomial, Theorem 3.4 provides an approximate rotation

$$
g^{i}:(U, y) \rightarrow\left(V, c_{0}(g)\right)
$$

with $d_{U}(y, z)=O(1)$ and in-radius $(U, z) \asymp|t|^{1-\varepsilon}$. For small $t, U$ contains a large neighborhood of $T$.

Let $T^{\prime}=g^{i}(T)$. Since $\operatorname{diam}(T) / d(T, \partial U) \asymp|t|^{\varepsilon}$, the Koebe distortion theorem shows

$$
\left|\sigma\left(T^{\prime}\right)-\sigma(T)\right|=O\left(|t|^{\varepsilon}\right)
$$

Let $T^{\prime \prime}=\phi\left(T^{\prime}\right)$. Since $\phi$ is $C^{1+\beta}$ at $c_{0}(g)$, we have

$$
\left|\sigma\left(T^{\prime \prime}\right)-\sigma\left(T^{\prime}\right)\right|=O\left(\frac{\left(d\left(T^{\prime}, c_{0}(g)\right)+\operatorname{diam}\left(T^{\prime}\right)\right)^{1+\beta}}{\operatorname{diam}\left(T^{\prime}\right)}\right) .
$$

Because $g \mid P(g)$ is quasiconformally conjugate to a rotation, there is a $\gamma>0$ such that $\operatorname{diam}\left(T^{\prime}\right)=O\left(\operatorname{diam}(T)^{\gamma}\right)=O\left(|t|^{\gamma}\right)$. Since $|t|^{\varepsilon} d(T, y)=O(\operatorname{diam}(T))$, Koebe again implies $|t|^{\varepsilon} d\left(T^{\prime}, c_{0}(g)\right)=O\left(\operatorname{diam}\left(T^{\prime}\right)\right)$. Combining these inequalities and choosing $\varepsilon$ suitably small, we obtain

$$
\left|\sigma\left(T^{\prime \prime}\right)-\sigma\left(T^{\prime}\right)\right|=O\left(|t|^{\beta \gamma-\varepsilon(1+\beta)}\right)=O\left(|t|^{\beta \gamma / 2}\right) .
$$

Finally consider $\phi(T)=h^{-i}\left(T^{\prime \prime}\right)$. Since $\phi$ is $K$-quasiconformal, from $\operatorname{diam}(T)=$ $O\left(|t|^{\varepsilon} d(T, \partial U)\right)$ we deduce $\operatorname{diam}(\phi(T))=O\left(|t|^{\eta} d(\phi(T), \partial \phi(U))\right)$, where $\eta=\varepsilon / K$. Applying Koebe once more to the univalent map $h^{i}: \phi(U) \rightarrow \phi(V)$, we conclude

$$
\left|\sigma(\phi(T))-\sigma\left(T^{\prime \prime}\right)\right|=O\left(|t|^{\eta}\right)
$$

All three bounds on the change in the similarity invariant are in terms of powers of $|t|$, so we obtain an $\alpha>0$ such that $|\sigma(\phi(T))-\sigma(T)|=O\left(|t|^{\alpha}\right)$. This establishes (5.2) and thereby completes the proof of Theorem 5.1.

\section{Renormalization}

In $\S 2$ we constructed renormalizations of rigid rotations on the circle. Since $f \mid \partial D$ is topologically conjugate to a rotation, it too admits such renormalizations.

In this section we show that the renormalizations of $f \mid \partial D$ can be complexified to yield holomorphic pairs $\left(f^{q_{n}}: U_{1} \rightarrow V, f^{q_{n+1}}: U_{2} \rightarrow V\right)$. These renormalizations are variants of the holomorphic commuting pairs introduced in $[\mathrm{F}]$. We then use the results of $\S 4$ to study the Lebesgue density of the escape loci for these renormalized dynamical systems. 


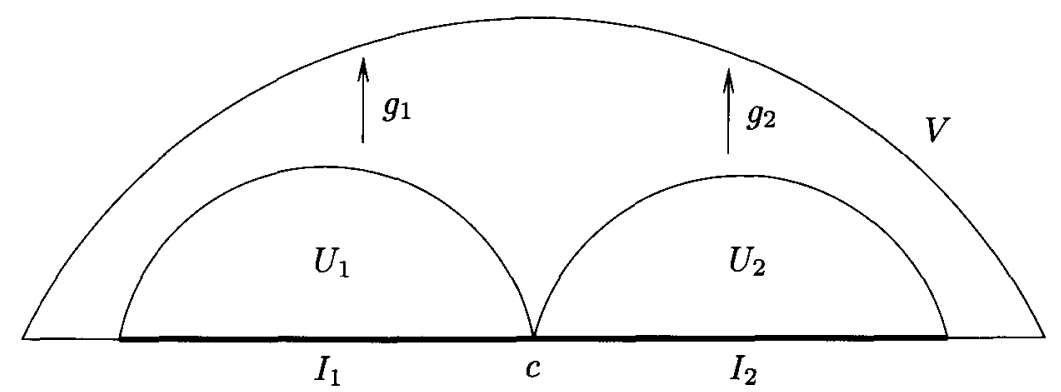

Fig. 10. A holomorphic pair

Holomorphic pairs. Let $g_{i}: U_{i} \rightarrow V, i=1,2$, be univalent maps between quasidisks in C with $U_{i} \subset V$. Each $g_{i}$ extends to a homeomorphism $\overline{U_{i}} \rightarrow \bar{V}$, which we denote by the same letter.

We say that $\left(g_{1}, g_{2}\right)$ is a holomorphic pair if (as in Figure 10)

(1) $V-\overline{U_{1} \cup U_{2}}$ is also a quasidisk;

(2) $\overline{U_{i}} \cap \partial V=I_{i}$ is an arc;

(3) $g_{i}\left(I_{i}\right) \subset I_{1} \cup I_{2}$, for $i=1,2$; and

(4) $\overline{U_{1}} \cap \overline{U_{2}}=\{c\}$ is a single point.

A holomorphic pair is designed to complexify the boundary dynamics of $\left(g_{1}, g_{2}\right)$ on $I_{1} \cup I_{2}$. Note that the interval $I_{1} \cup I_{2}$ inherits an orientation from $V$.

Let us say that a map between sets in $\mathbf{C}$ is quasisymmetric if it extends to a quasiconformal homeomorphism of the plane. We next show that boundary conjugacies can be promoted to the complex domain.

THEOREM 6.1. Let $\phi:\left(I_{1} \cup I_{2}\right) \rightarrow\left(I_{1}^{\prime} \cup I_{2}^{\prime}\right)$ be an orientation-preserving quasisymmetric map conjugating the boundary dynamics of one holomorphic pair $\left(g_{1}, g_{2}\right)$ to that of another $\left(g_{1}^{\prime}, g_{2}^{\prime}\right)$. Then $\phi$ extends to a quasiconformal conjugacy $\bar{\phi}: \bar{V} \rightarrow \bar{V}^{\prime}$.

Proof. Choose any extension of $\phi$ to a quasisymmetric map $\partial V \rightarrow \partial V^{\prime}$. Define $\phi$ on $\partial U_{i}$ by $\phi(x)=\left(g_{i}^{\prime}\right)^{-1} \circ \phi \circ g_{i}(x)$. This definition is consistent with the values already specified on $\partial U_{i} \cap \partial V=I_{i}$ by the conjugacy condition on $\phi$. An orientation-preserving quasisymmetric map between the boundaries of quasidisks extends to the interior, so we may further prolong $\phi$ to a quasiconformal map $\phi_{0}: \bar{V} \rightarrow \overline{V^{\prime}}$ which is a conjugacy on $\partial U_{1} \cup \partial U_{2}$.

Define $\phi_{1}: \bar{V} \rightarrow \overline{V^{\prime}}$ by

$$
\phi_{1}(x)= \begin{cases}\left(g_{i}^{\prime}\right)^{-1} \circ \phi_{0} \circ g_{i}(x) & \text { if } x \in U_{i}, \\ \phi_{0}(x) & \text { otherwise. }\end{cases}
$$

Then $\phi_{1}$ is quasiconformal, and it agrees with $\phi_{0}$ on $\partial V$. Therefore $\phi_{1}$ is isotopic to $\phi_{0}$ rel the ideal boundary of $V$. 
The map $\left(g_{1} \cup g_{2}\right):\left(U_{1} \cup U_{2}\right) \rightarrow V$ is a holomorphic covering, and in the language of the appendix, $\left(\phi_{0}, \phi_{1}\right)$ is a combinatorial conjugacy between $\left(g_{1} \cup g_{2}\right)$ and $\left(g_{1}^{\prime} \cup g_{2}^{\prime}\right)$. By Theorem A.1, there is a quasiconformal conjugacy $\bar{\phi}: \bar{V} \rightarrow \bar{V}^{\prime}$ with $\bar{\phi}=\phi_{0}=\phi$ on $\partial V$.

Siegel renormalization. Consider once again the quadratic polynomial $f(z)=$ $e^{2 \pi i \theta} z+z^{2}$, where $\theta$ has bounded type and $p_{n} / q_{n} \rightarrow \theta$ are its continued fraction approximants. Let $c_{-i}$ be the unique point in the boundary of the Siegel disk $D$ such that $f^{i}\left(c_{-i}\right)=c_{0}$

We say that $f$ is $\left(q_{n}, q_{n+1}\right)$-renormalizable if there is a holomorphic pair of the form

$$
\left(f^{q_{n}}: U_{1} \rightarrow V, f^{q_{n+1}}: U_{2} \rightarrow V\right)
$$

such that

(1) $V \cap D=\varnothing$,

(2) $\overline{U_{1}} \cap \overline{U_{2}}=c_{0}$, the critical point of $f$, and

(3) $I_{1}=\overline{U_{1}} \cap \partial V=\left[c_{r_{n}}, c_{0}\right]$, while $I_{2}=\overline{U_{2}} \cap \partial V=\left[c_{0}, c_{r_{n+1}}\right]$.

(As before, $[a, b] \subset \partial D$ denotes the interval of shorter length in the linear coordinate, and $\left.r_{n}=q_{n-1}-q_{n}.\right)$ The boundary dynamics of $\left(f^{q_{n}}, f^{q_{n+1}}\right)$ on $I_{1} \cup I_{2}$ agrees, in the linear coordinate, with the extended renormalization $\mathcal{R}_{n}(F)$ discussed at the end of $\S 2$.

THEOREM 6.2 (Siegel renormalizations). The map $f(z)=e^{2 \pi i \theta} z+z^{2}$ is $\left(q_{n}, q_{n+1}\right)$ renormalizable for every $n \geqslant 3$.

These renormalizations are conveniently constructed using external rays. To apply this method we need the following result from $[\mathrm{Pe}]$ :

Theorem 6.3 (Petersen). The Julia set $J(f)$ is locally connected.

By local connectivity, the Riemann mapping

$$
\omega:(\mathbf{C}-\vec{\Delta}) \rightarrow(\mathbf{C}-K(f))
$$

normalized so that $\omega\left(z^{2}\right)=f(\omega(z))$, extends continuously to a semiconjugacy $S^{1} \rightarrow J(f)$. The external rays $R_{t}$ and equipotentials $C_{s}$ are defined to be the images of the rays $\arg (z)=2 \pi t$ and the circles $|z|=\exp (2 \pi s)$ under $\omega$. (The term equipotential comes from the fact that $C_{s}$ is a level set for the Green function of $K(f)$.)

Proof of Theorem 6.2 (Siegel renormalizations). Let $J_{n}=\left[c_{0}, c_{r_{n}}\right] \subset \partial D$. The critical value $c_{1}$ is disjoint from $\overline{\bigcup_{n \geqslant 3} J_{n}}$.

Consider the annulus $A$ between the boundary of the Siegel disk and a fixed equipotential $C_{S}, S>0$. Let $R_{t}$ be the external ray landing at the critical value $c_{1}$. Let $V$ be 


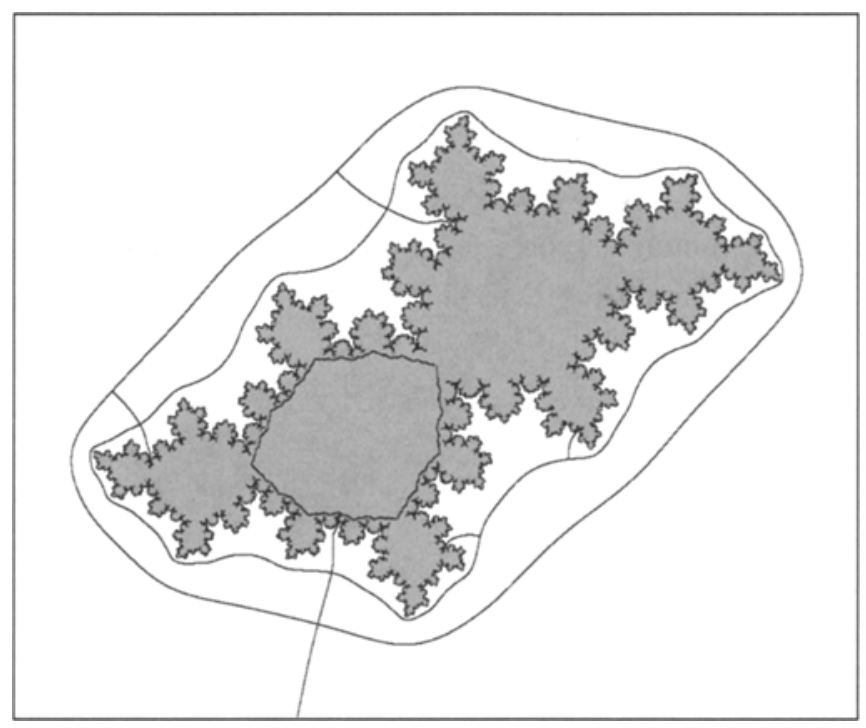

Fig. 11. Renormalization of the golden mean Siegel disk

a quasidisk obtained by removing from $A$ a slight thickening of $R_{t}$. The thickening need only be small enough that $\partial V$ is disjoint from every external ray landing in $\overline{\bigcup J_{n}}$.

The critical point $c_{0}$ lies in $\partial V$, but $V$ itself is disjoint from the postcritical set. Thus every component of $f^{-i}(V)$ is a quasidisk mapping univalently to $V$, and there is one component touching each element of $f^{-i}\left(c_{0}\right)$.

Let $U_{n}$ be the component of $f^{-q_{n}}(V)$ with $c_{-q_{n}} \in \partial U_{n}$. The critical values of $f^{i}$ are $\left\{c_{1}, c_{2}, \ldots, c_{i}\right\}$, so $\left[c_{q_{n}}, c_{q_{n-1}}\right]$ is the maximal interval around $c_{0}$ in $\partial D$ with no critical values of $f^{q_{n}}$ in its interior. We have $f^{q_{n}}\left(J_{n}\right)=\left[c_{q_{n}}, c_{q_{n-1}}\right]$ (by the definition of $r_{n}$ ), so $\overline{U_{n}} \cap \partial D=J_{n}$. Since $U_{n} \subset f^{-q_{n}}\left(A-R_{t}\right), U_{n}$ lies between a pair of external rays landing in $J_{n}$, and within the equipotential $C_{s}, s=2^{-q_{n}} S$. Therefore $\partial U_{n} \cap \partial V=\partial U_{n} \cap \partial D=J_{n}$.

By similar reasoning, $\overline{U_{n}}$ and $\overline{U_{n+1}}$ meet only at the critical point $c_{0}$. It is also easy to check that $V-\overline{U_{n} \cup U_{n+1}}$ is a quasidisk; for example, $\partial U_{n}$ and $\partial U_{n+1}$ meet nontangentially at the critical point because the quasidisk $D^{\prime}=\iota(D)$ comes between them. Thus the holomorphic pair

$$
\left(f^{q_{n}}: U_{n} \rightarrow V, f^{q_{n+1}}: U_{n+1} \rightarrow V\right)
$$

provides the desired renormalization of $f$.

Example. A $(3,5)$-renormalization of the golden mean Siegel disk is depicted in Figure 11. The external ray $R_{t}$ landing at the critical value $c_{1}$ enters at the bottom edge of the picture; it crosses the equipotentials $C_{2^{-3} S}$ and $C_{2^{-5} S}$, where $\exp (2 \pi S)=2$. (The equipotential $C_{S}$ is outside the frame.) Approximations to $U_{3}$ and $U_{5}$ appear to 


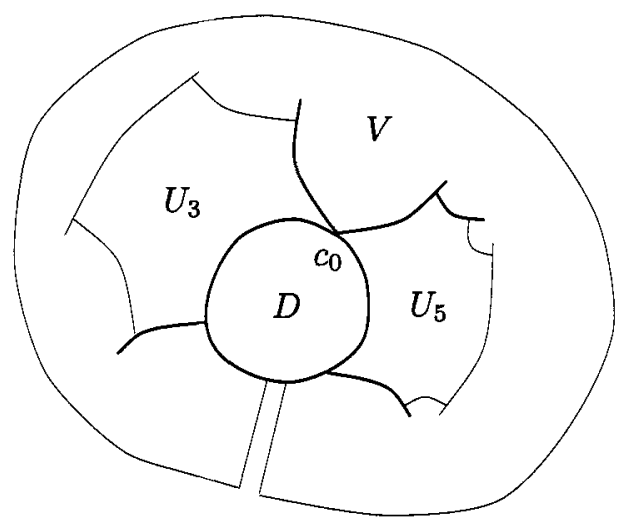

Fig. 12. Schematic of the renormalization

the left and right of the critical point respectively. The boundary of each approximate $U_{n}$ consists of external rays (landing at certain preimages of the critical point not on the boundary of the Siegel disk), several arcs running along preimages of $\partial D$, and the interval $J_{n}$ in $\partial D$. To obtain the actual renormalization, one must cut along slight thickenings of each external ray, as suggested in Figure 12.

Escape loci. Let $\left(g_{1}, g_{2}\right)$ be a holomorphic pair. Let $U=U_{1} \cup U_{2}$, and define

$$
g=\left(g_{1} \cup g_{2}\right): U \rightarrow V .
$$

By analogy with a polynomial-like map, we define the filled Julia set of $\left(g_{1}, g_{2}\right)$ by

$$
K\left(g_{1}, g_{2}\right)=\bigcap_{n>0} g^{-n}(U) .
$$

If $\left(g_{1}, g_{2}\right)$ is a renormalization of $f$, then $K\left(g_{1}, g_{2}\right) \subset J(f)$, because under iteration these points stay bound, but they never reach the Siegel disk $D$. In particular, the filled Julia set of a renormalization is nowhere dense and of measure zero.

It is also useful to include points which escape from $U$ but do so by landing within distance $\varepsilon$ of the "critical point" $\{c\}=\overline{U_{1}} \cap \overline{U_{2}}$. To this end we define

$$
K_{\varepsilon}\left(g_{1}, g_{2}\right)=K\left(g_{1}, g_{2}\right) \cup \bigcup_{n>0} g^{-n}(B(c, \varepsilon)-U) .
$$

Proposition 6.4. For any renormalization

$$
\left(f^{q_{n}}: U_{n} \rightarrow V, f^{q_{n+1}}: U_{n+1} \rightarrow V\right)
$$

of $f$, the critical point $c_{0}$ lies in the interior of $\bar{D} \cup \overline{D^{\prime}} \cup U_{1} \cup U_{2}$.

Proof. Since $f^{q_{n}}$ and $f^{q_{n+1}}$ each map $c_{0}$ into the interior of the arc $\partial V \cap \partial D$, a neighborhood of $c$ in $\partial\left(U_{1} \cup U_{2}\right)$ is contained in $\partial\left(D \cup D^{\prime}\right)$. 
COROllary 6.5. For all $\varepsilon>0$ sufficiently small, there is a $\delta>0$ such that

$$
K_{\delta}(f) \cap\left(U_{1} \cup U_{2}\right) \subset K_{\varepsilon}\left(f^{q_{n}}, f^{q_{n+1}}\right) \subset K(f) .
$$

Proof. Let $U=U_{1} \cup U_{2}$. For $\varepsilon$ small enough, the part of $V-U$ within distance $\varepsilon$ of $c=c_{0}$ lies in the pre-Siegel disk $\bar{D}^{\prime}$. Thus points which escape from $U$ by landing in $B(c, \varepsilon)$ must also lie in $K(f)$.

Now consider a point $z \in K_{\delta}(f) \cap U$ that escapes under iteration of the renormalized map, by landing at $z^{\prime} \in V-U$. By assumption $z$ remains close to $\partial D$, and thus close to $I_{1} \cup I_{2}$, until the moment it escapes. Thus $z^{\prime}$ is close to $f^{q_{n}}\left(I_{1}\right) \cup f^{q_{n+1}}\left(I_{2}\right)=J$. But $J$ is contained in the interior of $I_{1} \cup I_{2}$, so $\overline{V-U} \cap J=\{c\}$, and thus for $\delta$ sufficiently small we must have $z^{\prime} \in B(c, \varepsilon)$.

COROLlaRY 6.6. The critical point $c_{0}$ is a measurable deep point of

$$
D \cup D^{\prime} \cup K_{\varepsilon}\left(f^{q_{n}}, f^{q_{n+1}}\right)
$$

for every $\varepsilon>0$.

Proof. By Corollary 4.5, $c_{0}$ is a measurable deep point of $K_{\delta}(f)$.

\section{Self-similarity}

In this section we show that the Siegel disk of $f(z)=e^{2 \pi i \theta} z+z^{2}$ is self-similar when $\theta$ is a quadratic irrational.

A succinct description of this self-similarity can be formulated as follows. Suppose that $\theta=\left[a_{1}, a_{2}, \ldots\right]$, where $a_{n+s}=a_{n}$ for all $n \geqslant N$. Let $F(x)=x+\theta$ be the standard rotation on $S^{1}=\mathbf{R} / \mathbf{Z}$. By Corollary 2.2 there is a $\gamma \in(0,1)$ such that near $x=0$, the contraction $\tilde{\psi}(x)=(-1)^{s} \gamma x$ conjugates $F^{q_{n}}$ to $F^{q_{n+s}}$ for $n \gg 0$. The topological conjugacy from $\left\langle S^{1}, F\right\rangle$ to $\langle\partial D, f\rangle$, normalized to send $x=0$ to the critical point, transports $\tilde{\psi}$ to a quasisymmetric mapping $\psi$ defined on a neighborhood of $c_{0}$ in $\partial D$. Then we have

THEOREM 7.1 (Self-similarity). The mapping $\psi$ is $C^{1+\alpha}$-conformal or anticonformal at the critical point. That is, for all $z$ in a neighborhood of $c_{0}$ in $\partial D$, we have

$$
\psi(z)= \begin{cases}c_{0}+\lambda\left(z-c_{0}\right)+O\left(\left|z-c_{0}\right|^{\alpha}\right), & \text { for } s \text { even, or } \\ c_{0}+\lambda\left(\overline{z-c_{0}}\right)+O\left(\left|z-c_{0}\right|^{\alpha}\right), & \text { for } s \text { odd, }\end{cases}
$$

where $\alpha>0, \lambda \in \mathbf{C}$ and $0<|\lambda|<1$.

Here is a more complete statement which extends $\psi$ to a conjugacy between holomorphic pairs. By results of the preceding section, we may choose a sequence of renormalizations

$$
\left(f^{q_{n}}: U_{1}^{n} \rightarrow V^{n}, f^{q_{n+1}}: U_{2}^{n} \rightarrow V^{n}\right)
$$


for all $n \geqslant 3$.

THEOREM 7.2. For any $n \geqslant \max (N, 3)$, there exists a homeomorphism

$$
\psi: \overline{V^{n}} \rightarrow \overline{V^{n+s}},
$$

fixing the critical point $c_{0}$ and conjugating the $\left(q_{n}, q_{n+1}\right)$-renormalization of $f$ to its $\left(q_{n+s}, q_{n+s+1}\right)$-renormalization. For even $s$, the conjugacy can be chosen so that:

(1) $\psi$ extends to a quasiconformal map on $\overline{V^{n}} \cup \bar{D}$, conformal on a neighborhood of $c_{0}$ in $D$;

(2) the extended mapping is $C^{1+\alpha}$-conformal at the critical point; and

(3) $\psi^{\prime}\left(c_{0}\right)$ is independent of $n$ and satisfies $0<\left|\psi^{\prime}\left(c_{0}\right)\right|<1$.

For odd $s, \psi$ is orientation-reversing, and the same statements hold for $\overline{\psi(z)}$.

Proof. The idea is the same as that used in the proof of universality: a conjugacy between two successive renormalizations is smooth at the critical point because it is conformal on a set of high density.

We first assume that $s$ is even.

Let $U^{n}=U_{1}^{n} \cup U_{2}^{n}$, and let $L_{n}=\overline{U^{n}} \cap \partial D=\left[c_{r_{n}}, c_{r_{n+1}}\right]$. Consider the dynamics in the linear coordinate system $\mathbf{C}_{w}$. On the disk $\{w:|w| \leqslant 1\}$, we have $f(w)=F(w)=e^{2 \pi i \theta} w$. The $\operatorname{arc} L_{n}$ is contained in the circle $|w|=1$, and the boundary dynamics of $\left(f^{q_{n}}, f^{q_{n+1}}\right)$ is the same as that of the extended renormalization $\mathcal{R}_{n}(F)$ discussed in $\S 2$. By Corollary 2.2 , there is a $\gamma \in(0,1)$ (depending only on $\theta$ ) such that the map $\phi: L_{n} \rightarrow L_{n+s}$ given by $\phi(w)=w^{\gamma}$ conjugates $\mathcal{R}_{n}(F)$ to $\mathcal{R}_{n+s}(F)$. (Here $\phi$ is normalized to fix the critical point, so $\phi(1)=1$.)

Since the linearizing map $\Phi: \mathbf{C}_{z} \rightarrow \mathbf{C}_{w}$ is quasiconformal, $\phi$ is quasisymmetric in the original coordinate $\mathbf{C}_{z}$. By Theorem $6.1, \phi$ extends to a quasiconformal conjugacy $\phi_{0}: \overline{V^{n}} \rightarrow \overline{V^{n+s}}$

Next we modify this conjugacy so that it is conformal on a neighborhood of the critical point in $\overline{D \cup \overline{D^{\prime}}}$. Since $\Phi \mid D$ is conformal, there is a quasiconformal map $\beta: D \rightarrow D$, prolonging $\phi_{0}$ on $\partial V \cap \partial D$, such that $\beta(z)$ is conformal on a neighborhood of the critical point. Indeed, near $c_{0}$ we may simply take $\beta(z)=\Phi^{-1}\left(\Phi(z)^{\gamma}\right)$. Conjugating by the involution $\iota: D \rightarrow D^{\prime}$, we obtain a map $\beta^{\prime}: D^{\prime} \rightarrow D^{\prime}$ which is conformal near $c_{0}$ and agrees with $\phi_{0}$ on a neighborhood of $c_{0}$ in $\partial\left(V^{n}-U^{n}\right)$. Thus we may modify $\phi_{0}$ to a quasiconformal map $\phi_{1}: \overline{V^{n}} \rightarrow \overline{V^{n+s}}$ such that

(1) $\phi_{1}(z)=\phi_{0}(z)$ on $\overline{U^{n}} \cup \partial V_{n}$; and

(2) $\phi_{1}(z)=\beta^{\prime}(z)$ on a neighborhood of $c_{0}$ in $V^{n}-\breve{U}^{n}$.

The pair $\left(\phi_{0}, \phi_{1}\right)$ is a combinatorial conjugacy between the two renormalizations, in the sense of the Appendix. By Theorem A.1, there is a quasiconformal conjugacy

$$
\psi: \overline{V^{n}} \rightarrow \overline{V^{n+s}},
$$




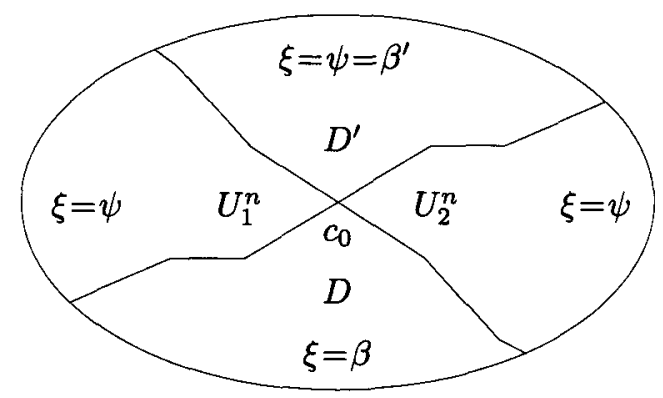

Fig. 13. A neighborhood of the critical point

equal to $\phi_{1}$ on $V^{n}-U^{n}$. In particular, $\psi$ is conformal on $B\left(c_{0}, \varepsilon\right) \cap(V-\bar{U})$ for some $\varepsilon>0$.

The points of conformality are invariant under the dynamics, so $\psi$ is also conformal a.e. on $K_{\varepsilon}\left(f^{q_{n}}, f^{q_{n+1}}\right)$. (Since $K\left(f^{q_{n}}, f^{q_{n+1}}\right) \subset J(f)$ has measure zero, almost every point in $K_{\varepsilon}\left(f^{q_{n}}, f^{q_{n+1}}\right)$ escapes through $B(c, \varepsilon)-U^{n}$.)

The extended map

$$
\xi=\psi \cup \beta: \overline{V^{n}} \cup D \rightarrow \overline{V^{n+s}} \cup D
$$

is conformal on

$$
E=K_{\varepsilon}\left(f^{q_{n}}, f^{q_{n+1}}\right) \cup\left(B\left(c_{0}, \varepsilon\right) \cap\left(D \cup D^{\prime}\right)\right)
$$

(see Figure 13). By Corollary 6.6, $c_{0}$ is a measurable deep point of $E$, so $\xi$ is $C^{1+\alpha_{-}}$ conformal at the critical point, by the Boundary Conformality Theorem 5.4.

Since in the linear coordinate, $\psi \mid S^{1}$ is a contraction by $\gamma, 0<\gamma<1$, we have that $0<\left|\psi^{\prime}\left(c_{0}\right)\right|<1$. The germ of $\psi \mid \partial D$ at $c_{0}$ is independent of $n$, so $\psi^{\prime}\left(c_{0}\right)$ is also independent of $n$.

Finally, when $s$ is odd the map $\phi: L_{n} \rightarrow L_{n+s}$ conjugating $\mathcal{R}_{n}(F)$ to $\mathcal{R}_{n+s}(F)$ has the form $\phi(w)=\bar{w}^{\gamma}$. Thus we may promote $\overline{\phi(z)}$ to a quasiconformal conjugacy $\overline{\psi(z)}$ between $\left(f^{q_{n}}(z), f^{q_{n+1}}(z)\right)$ and the complex conjugate dynamical system $\left(\overline{f^{q_{n+s}}(\bar{z})}, \overline{f^{q_{n+s+1}}(\bar{z})}\right)$. Then $\overline{\psi(z)}$ has the same properties enjoyed by $\psi(z)$ for $s$ even.

Let

$$
A(z)= \begin{cases}c_{0}+\frac{\partial \psi}{\partial z}\left(c_{0}\right)\left(z-c_{0}\right), & s \text { even } \\ c_{0}+\frac{\partial \psi}{\partial \bar{z}}\left(c_{0}\right)\left(\overline{z-c_{0}}\right), & s \text { odd }\end{cases}
$$

denote the linearization of $\psi$ at the critical point.

CoROllary 7.3. The blowups $A^{-n}(\partial D)$ of the boundary of the Siegel disk converge exponentially fast to an A-invariant quasicircle through $\infty$, in the Hausdorff metric on compact subsets of the sphere. 
Proof. Fix any conjugacy $\psi$ as in the theorem and let $\lambda=\left|\psi^{\prime}\left(c_{0}\right)\right|$ or $\left|\bar{\psi}^{\prime}\left(c_{0}\right)\right|$, depending on whether $s$ is even or odd. Then $\lambda<1$. For $n \gg 0$ choose a segment $I \subset \partial D$ of diameter about $\lambda^{n}$ such that $A^{-n} I$ is the component of $A^{-n}(\partial D) \cap B\left(c_{0}, 1\right)$ passing through the critical point. Then $\psi(I) \subset \partial D$ and since $\psi$ is $C^{1+\alpha}$, the Hausdorff distance $d_{H}$ satisfies

$$
d_{H}\left(A^{-n} I, A^{-(n+1)} \psi(I)\right) \leqslant \lambda^{-n} d_{H}\left(I, A^{-1} \psi(I)\right)=O\left(\lambda^{-n}(\operatorname{diam} I)^{1+\alpha}\right)=O\left(\lambda^{\alpha n}\right) .
$$

Thus the blowups of $\partial D$ converge exponentially fast at scale 1 around the critical point. A similar argument holds on the sphere.

Using the fact that $\psi\left(c_{q_{n}}\right)=c_{q_{n+s}}$ for all $n \gg 0$, it is also easy to verify

COROLLARY 7.4. There exist $d_{0}, \ldots, d_{s-1} \in \mathbf{C}$ such that the closest returns of the critical orbit satisfy

$$
c_{q_{n}}=A^{m}\left(d_{i}\right)+O\left(\left|c_{0}-c_{q_{n}}\right|^{1+\alpha}\right),
$$

where $n=m s+i$.

Spiraling. A Jordan curve $C$ in the plane spirals about $c \in C$ if any continuous branch of $\arg (z-c)$ on $C-\{c\}$ is unbounded. The curve $C$ does not spiral if and only if there exists an $n$ such that one petal of $\sqrt[n]{C-c}$ lies in a half-plane.

CoROLLARY 7.5. If the period $s$ is odd, then the boundary of the Siegel disk does not spiral about the critical point.

Proof. It suffices to show that the Hausdorff limit $X=\lim A^{-n}(\partial D)$ does not spiral about $c_{0}$. Pick any point $p$ in the limiting disk $D_{\infty}=\operatorname{int} \lim A^{-n}(\bar{D})$, and let $\gamma \subset D_{\infty}$ be a path joining $p$ to $A(p)$. Then

$$
\delta=\gamma \cup A(\gamma) \cup A^{2}(\gamma) \cup \ldots
$$

converges to $c_{0}$ without spiraling, since alternate segments wind in opposite directions. Since $\delta$ is disjoint from $X$, the latter does not spiral either.

\section{The limiting dynamics}

In this section we describe more completely the limiting dynamical system obtained by rescaling the first return maps around the critical point. As in the preceding section, $f(z)=e^{2 \pi i \theta} z+z^{2}$, where $\theta=\left[a_{1}, a_{2}, \ldots\right]$ and $a_{i}=a_{i+s}$ for $i \gg 0$.

Let $b_{i}, i \in \mathbf{Z}$, be the unique sequence with period $s$ that agrees with $a_{i}$ for $i \gg 0$. Let $\theta_{i}=\left[b_{i}, b_{i+1}, \ldots\right]$, and define $t_{i}$ so that $t_{0}=\theta_{1}$ and $t_{i}=-\theta_{i+1} t_{i-1}$.

Let $A(z)$ be the linearization of the contracting self-similarity map given by (7.1). Let $D_{\infty} \subset \mathbf{C}$ denote limit of the rescaled Siegel disks; it is the unique quasidisk such that $A^{-n}(\bar{D}) \rightarrow \overline{D_{\infty}}$ in the Hausdorff topology. 
THEOREM 8.1 (Limiting dynamics). There exists a sequence of analytic functions $g_{i}: W_{i} \rightarrow \mathbf{C}, i \in \mathbf{Z}$, such that

(1) $W_{i}$ is an open disk with $\overline{D_{\infty}} \subset W_{i} \subset \mathbf{C}$;

(2) $\partial W_{i}$ is the natural boundary of $g_{i}$;

(3) $g_{i}$ is a $\sigma$-proper branched covering of the plane;

(4) $g_{i}(z)=\lim _{n \rightarrow \infty} A^{-n} \circ f^{q_{n s+i}} \circ A^{n}(z)$;

(5) on each compact subset of $W_{i}$, this limit converges uniformly and exponentially fast;

(6) for all $z \in W_{i}, g_{i}(z)=g_{i-1}^{b_{i}} \circ g_{i-2}(z)=A^{-1} \circ g_{i+s} \circ A(z)$; and

(7) there is a Riemann mapping $D_{\infty} \rightarrow \mathbf{H}$ which conjugates the mappings $\left\langle g_{i}(z)\right\rangle$ to the translations $\left\langle T_{i}(z)=z+t_{i}\right\rangle$.

Remark on universality. By the results of $\S 5$, any quadratic-like map $h$ with a fixed point of multiplier $e^{2 \pi i \theta}$ is $C^{1+\alpha}$-conjugate to $f$ on the boundary of its Siegel disk. Thus $P(h)$ is self-similar about $c_{0}(h)$ with the same rescaling factor as $P(f)$, Corollaries 7.3, 7.4 and 7.5 also hold for $h$, and the rescalings of $h^{q_{n}}$ converge to the same dynamical system $\left\langle g_{i}\right\rangle$ as above.

Before embarking on the proof a few preliminaries are in order.

Branched coverings and $\sigma$-proper maps. Let $g: X \rightarrow Y$ be a holomorphic map between Riemann surfaces. Then $g$ is:

(1) proper, if $g^{-1}(K)$ is compact for each compact $K \subset Y$;

(2) $\sigma$-proper, if each component of $g^{-1}(K)$ is compact for each compact $K \subset Y$; and

(3) a branched covering, if each $y \in Y$ has a neighborhood $V$ such that $g: U \rightarrow V$ is proper for each component $U$ of $g^{-1}(V)$.

It is not hard to show that each condition implies the next. Also, $g$ is $\sigma$-proper if and only if $X$ and $Y$ can be expressed as increasing unions of subsurfaces $X_{i}, Y_{i}$ such that $g: X_{i} \rightarrow Y_{i}$ is proper (hence the terminology). The trigonometric function $\sin : \mathbf{C} \rightarrow \mathbf{C}$ is a branched covering which is not $\sigma$-proper, because $\sin ^{-1}([-1,1])=\mathbf{R}$ is not compact. The maximal analytic continuation $\widetilde{F}$ of the Feigenbaum fixed-point, on the other hand, is a $\sigma$-proper branched covering of the plane (cf. [Mc4, §7]). The composition of two $\sigma$-proper maps is also $\sigma$-proper; this property fails for branched coverings.

Proposition 8.2. Let $g: U \rightarrow \mathbf{C}$ be a branched covering. Then every $p \in \partial U$ is a singular point of $g$.

Proof. Suppose to the contrary that there exists a $p \in \partial U$ and an analytic function $h$ extending $g$ near $p$. Then the branched covering condition implies that $g^{-1}(h(p))$ accumulates on $p$, so $h$ is constant, which is impossible. 
Carathéodory convergence. A sequence of pointed open disks $\left(U_{n}, u_{n}\right)$ in $\widehat{\mathbf{C}}$ converges to $(U, u)$ in the Carathéodory topology if $u_{n} \rightarrow u$, and if for any Hausdorff limit $K=$ $\lim \left(\widehat{\mathbf{C}}-U_{n_{k}}\right), U$ is the component of $\widehat{\mathbf{C}}-K$ containing $u$.

THEOREM 8.3 (Limits of proper maps). Let

$$
g_{n}:\left(U_{n}, u_{n}\right) \rightarrow\left(V_{n}, v_{n}\right)
$$

be a sequence of proper maps between pointed disks, with $\operatorname{deg}\left(g_{n}\right) \leqslant d$. Suppose that $u_{n} \rightarrow u$, that $g_{n}$ converges uniformly to a nonconstant limit on a neighborhood of $u$, and that $\left(V_{n}, v_{n}\right) \rightarrow(V, v)$. Then $\left(U_{n}, u_{n}\right)$ converges to a pointed disk $(U, u)$, and $g_{n}$ converges uniformly on compact subsets of $U$ to a proper map

$$
g:(U, u) \rightarrow(V, v)
$$

with $1 \leqslant \operatorname{deg}(g) \leqslant d$.

Proof. By [Mc3, Theorems 5.2 and 5.6], we can obtain convergence of $\left(U_{n}, u_{n}\right)$ and $g_{n}$ after passing to a subsequence. But any two subsequences have the same limit, by convergence near $u$.

Harmonic measure and exponentially fast convergence. Let $E \subset X$ be a closed subset of a Riemann surface. The harmonic measure $\omega_{E}(z)$ is the probability that a Brownian path starting at $z$ will land in $E$ before exiting $X$. It is a harmonic function on $X-E$. If $\omega_{E}(z)>0$ then $E$ has positive harmonic measure. The "two-constant theorem" controls a bounded holomorphic function on $X$ in terms of its behavior on $E$.

THEOREM 8.4. Let $f: X \rightarrow \mathbf{C}$ be an analytic function, with $|f(z)|$ bounded by $M$ on $X$ and by $m$ on $E$. Then $|f(z)| \leqslant M^{1-\delta} m^{\delta}$ on $\left\{z: \omega_{E}(z) \geqslant \delta\right\}$.

See [Ah2, Theorem 3-2].

Let us say that $f_{n} \rightarrow f$ exponentially fast on $E$ if there exist $C$ and $\lambda<1$ such that

$$
\sup _{E}\left|f_{n}(z)-f(z)\right|<C \lambda^{n} .
$$

COROLlaRY 8.5. Let $f$ and $\left\langle f_{n}\right\rangle$ be analytic functions, uniformly bounded on compact subsets of $X$, and suppose that $f_{n} \rightarrow f$ exponentially fast on a set $E \subset X$ of positive harmonic measure. Then $f_{n} \rightarrow f$ exponentially fast on any compact subset of $X$.

Of course the $C$ and $\lambda$ depend on the compact set in question.

Proof of Theorem 8.1 (Limiting dynamics). To simplify notation, we will discuss the branched covering $g_{0}: W_{0} \rightarrow \mathbf{C}$ in detail, the case of $g_{i}$ being analogous. 
Let $\psi: \bar{D} \rightarrow \bar{D}$ be a self-similarity homeomorphism of the type provided by Theorem 7.2. That is, for $n \gg 0, \psi$ conjugates $f^{q_{n}}$ to $f^{q_{n+s}}$ on a neighborhood of $c_{0}$, and

$$
\psi(z)=A(z)+O\left(\left|z-c_{0}\right|^{1+\alpha}\right) .
$$

We first show that for $z \in D_{\infty}$,

$$
\lim A^{-n_{\circ}} f^{q_{n s} \circ} A^{n}(z)
$$

exists, and the convergence is exponentially fast on compact subsets of $D_{\infty}$. Let $D_{n}=$ $A^{-n}(D)$ denote the $n$th dilate of the Siegel disk, and let

$$
f_{0, n}=A^{-n_{\mathrm{c}}} f^{q_{n s} \circ} A^{n}: \overline{D_{n}} \rightarrow \overline{D_{n}} .
$$

Then

$$
\psi_{n}=A^{-n_{\circ}}\left(A^{-1} \circ \psi\right) \circ A^{n}: \overline{D_{n}} \rightarrow \overline{D_{n+1}}
$$

conjugates $f_{0, n}$ to $f_{0, n+1}$ on a definite neighborhood of $c_{0}$; on the other hand, $\psi_{n}$ converges to the identity exponentially fast by (8.1). Thus $\left\langle f_{0, n}(z)\right\rangle$ converges exponentially fast on some compact ball $B \subset D_{\infty}$.

Now each $f_{0, n}$ is a conformal rotation of a quasidisk, so it can be extended to a $K_{0}$-quasiconformal map of the plane, where $K_{0}$ is independent of $n$. By convergence of $f_{0, n} \mid B$, the sequence $\left\langle f_{0, n}\right\rangle$ is bounded and equicontinuous on compact subsets of $D_{\infty}$. By Corollary 8.5 above, $\left\langle f_{0, n}\right\rangle$ converges exponentially fast on compact subsets of $D_{\infty}$.

To proceed further we need some additional notation. For $i \in \mathbf{Z}$, let

$$
d_{i}=\lim A^{-n}\left(c_{q_{n s+i}}\right) \in \partial D_{\infty}
$$

denote the limiting position of the rescaled closest returns of the critical point (cf. Corollary 7.4). Then $d_{i+s}=A\left(d_{i}\right)$, and $\left\{d_{i}: i \in \mathbf{Z}\right\}$ is a finite union of geometric sequences converging to the critical point.

Just as the boundary of the Siegel disk divides $\mathbf{C}$ into two domains, $D$ and $\Omega$, the quasiarc $\partial D_{\infty}$ divides $\mathbf{C}$ into two domains, $D_{\infty}$ and $\Omega_{\infty}$. Mimicking the notation $\left[c_{i}, c_{j}\right] \subset \partial D$, we let $\left[d_{i}, d_{j}\right] \subset \partial D_{\infty}$ denote the unique quasiarc in $\partial D_{\infty}$ with endpoints $d_{i}$ and $d_{j}$.

We now extend the domain of convergence of $A^{-n_{\circ}} f^{q_{n s}} \circ A^{n}$ beyond $D_{\infty}$. Let $\Omega^{\prime}=$ $\Omega-S$, where $S$ is an arc (e.g. an external ray) connecting $c_{1}$ to $\infty$ through $\Omega$. By cutting along $S$ we make $\Omega$ simply-connected. Consider the open disk

$$
V_{k, n}=\operatorname{int} A^{-n}\left(D \cup \Omega^{\prime} \cup\left[c_{q_{n s-k}}, c_{q_{n s-k-1}}\right]\right),
$$


obtained by joining $D$ and $\Omega^{\prime}$ together along an open arc in $\partial D$, then rescaling. Note that $V_{k, n} \supset D_{n}$. Picking basepoints $p_{n} \in D_{n}$ converging to $p \in D_{\infty}$, we have

$$
\left(V_{k, n}, p_{n}\right) \rightarrow\left(V_{k}, p\right)=\operatorname{int}\left(D_{\infty} \cup \Omega_{\infty} \cup\left[d_{-k}, d_{-k-1}\right]\right)
$$

in the Carathéodory topology. Note that the slit $S$ moves off to infinity and disappears in the limit. There is a unique disk $U_{k, n} \supset D_{n}$ such that

$$
f_{k, n}=A^{-n} \circ f^{q_{n s}} \circ A^{n}: U_{k, n} \rightarrow V_{k, n}
$$

is a proper map. (The disk $U_{k, n}$ is a component of the preimage of $V_{k, n}$ under the map $A^{-n_{\circ}} f^{q_{n s} \circ} A^{n}$.)

We claim that for fixed $k$, the degree of the proper map $f_{n, k}$ is eventually constant as $n \rightarrow \infty$. Indeed, the domain and range of $f_{k, n}$ are simply-connected, so $\operatorname{deg}\left(f_{k, n}\right)$ is one more than the number of its critical values. But the critical values of $f_{k, n}$ correspond to a subset of the critical values $\left\{c_{1}, c_{2}, \ldots, c_{q_{n s}}\right\}$ of $f^{q_{n s}}$, namely those lying in the interior of the arc $\left[c_{q_{n s-k}}, c_{q_{n-k-1}}\right]$. Since the continued fraction expansion of $\theta$ is preperiodic, this subset has constant cardinality for all $n$ sufficiently large, and thus $\operatorname{deg}\left(f_{k, n}\right)$ is also eventually constant.

Now we already have convergence of $f_{k, n} \mid D_{n}$ as $n \rightarrow \infty$, so by Theorem 8.3 there is a pointed disk $\left(U_{k}, p\right)$ and a proper map

$$
f_{k}:\left(U_{k}, p\right) \rightarrow\left(V_{k}, p\right)
$$

such that $f_{k, n} \rightarrow f_{k}$ uniformly on compact sets of $U_{k}$ as $n \rightarrow \infty$. Each of these limits extends the domain of convergence of $A^{-n} \circ f^{q_{n s}} \circ A^{n}$.

Clearly $V_{k, n} \subset V_{k+1, n}$ and thus $V_{k} \subset V_{k+1}$. Similarly $U_{k} \subset U_{k+1}$ and $f_{k}=f_{k+1} \mid U_{k}$. Letting $W_{0}=\bigcup U_{k}$, and noting $\mathbf{C}=\bigcup V_{k}$, we obtain a branched covering

$$
g_{0}: W_{0} \rightarrow \mathbf{C}
$$

by setting $g_{0}=\bigcup f_{k}$. Since each $f_{k}$ is proper, the map $g_{0}$ is $\sigma$-proper, and by Proposition $8.2, \partial W_{0}$ is its natural boundary. Since $\overline{D_{\infty}} \subset \bigcup V_{k}$, we also have $\overline{D_{\infty}} \subset W_{0}$. Finally we have shown above that $A^{-n_{\circ}} f^{q_{n s}} \circ A^{n} \rightarrow g_{0}$ exponentially fast on compact subsets of $D_{\infty}$, so by Corollary 8.5 the convergence is also exponentially fast on compact subsets of $W_{0}$.

This completes the proof of statements (1)-(5) of Theorem 8.1. Statement (7) follows easily by a linear computation similar to Theorem 2.1 .

To verify (6), we must show that $g_{i-1}^{b_{i}} \circ g_{i-2}$ and $A^{-1} \circ g_{i+s} \circ A$ are defined on $W_{i}$. First note that

$$
g_{i}=g_{i-1}^{b_{i}} \circ g_{i-2}
$$


when restricted to $D^{\infty}$. Since each $g_{i}$ is $\sigma$-proper, the composition on the right defines a branched covering of the plane, as does the map on the left. A branched covering of the plane admits no analytic continuation, so the domains of these two mappings must agree, and thus equality holds on $W_{i}$.

The same principle establishes the rescaling equation $g_{i}=A^{-1} \circ g_{i+s} \circ A$.

\section{A. Appendix: Promoting combinatorial equivalence to conjugacy}

Let $X=\Delta / \Gamma$ be a hyperbolic Riemann surface, presented as the quotient of the unit disk by a Fuchsian group $\Gamma$. Let $\Omega \subset S^{1}$ be the complement of the limit set of $\Gamma$. Then $(\Delta \cup \Omega) / \Gamma$ is a surface with boundary $\Omega / \Gamma$, the ideal boundary of $X$.

Any quasiconformal homeomorphism between hyperbolic surfaces extends to the ideal boundary.

Let $X_{1} \subset X_{0}$ be a pair of complex 1-manifolds (perhaps disconnected) with each component hyperbolic, and let $f: X_{1} \rightarrow X_{0}$ be a holomorphic covering map. A combinatorial equivalence from $f$ to another such dynamical system $g: Y_{1} \rightarrow Y_{0}$ is a pair of quasiconformal maps $\phi_{i}: X_{0} \rightarrow Y_{0}, i=0,1$, such that

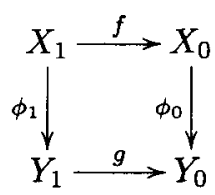

commutes, and $\phi_{0}$ is isotopic to $\phi_{1}$ rel the ideal boundary of $X_{0}$. If $\phi_{0}=\phi_{1}$ then $\phi_{0}$ is a quasiconformal conjugacy.

Theorem A.1 (Combinatorial promotion). Any combinatorial equivalence $\left(\phi_{0}, \phi_{1}\right)$ is isotopic, rel ideal boundary, to a quasiconformal conjugacy $\psi$. Moreover, $\psi$ can be chosen to agree with $\phi_{1}$ on $X_{0}-X_{1}$.

Proof. The proof relies on some basic facts about natural extensions and quasiconformal isotopies treated in detail in [DE] and [EMc].

Using [EMc, Theorem 1.3 and Proposition 2.3], the initial isotopy $\phi:[0,1] \times X_{0} \rightarrow Y_{0}$ may be chosen to be uniformly continuous in the hyperbolic metric. That is, we can arrange that

$$
d_{Y_{0}}\left(\phi_{t}(x), \phi_{s}(x)\right) \leqslant \delta(|s-t|),
$$

where $\delta(r) \rightarrow 0$ as $r \rightarrow 0$, and distance is measured along the geodesic arc homotopic to the trace of the isotopy. 
Roughly speaking, $\phi_{1}$ gives a map on the "fundamental domain" $X_{0}-X_{1}$, which is a conjugacy on the ideal boundary, so it can be pulled back repeatedly to obtain conjugacies on larger and larger regions. In the limit one obtains a conjugacy outside a region $\Omega$ mapped to itself by $f$. A canonical representative of the map on $\Omega$ will automatically commute with coverings, and give a conjugacy there.

More formally, let $X_{n}=f^{-n}\left(X_{0}\right)$ and $Y_{n}=g^{-n}\left(Y_{0}\right)$. Then there is a unique extension of $\phi_{t}$ to an isotopy defined for all $t \in[0, \infty)$ such that

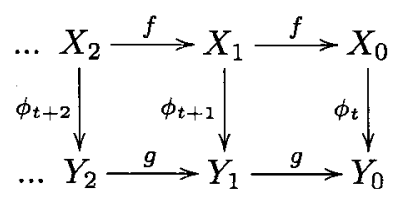

commutes for $t \in[0,1]$, and $\phi_{t}(x)=\phi_{n}(x)$ for $t>n$ and $x \notin X_{n}$. Here we use the fact that the hyperbolic metric on $X_{n}$ blows up at $\partial X_{n}$ to insure that $\phi_{t}(x)$ and $\phi_{n}(x)$ agree there for $t \in[n, n+1]$. Indeed, we have

$$
d_{Y_{n}}\left(\phi_{t}(x), \phi_{s}(x)\right) \leqslant \delta(|s-t|)
$$

for $x \in X_{n}$ and $s, t \geqslant n$, because the covering maps $f$ and $g$ are local hyperbolic isometries. Since inclusions are contracting, (A.1) also holds for all $s, t \geqslant 0$ and $x \in X_{0}$.

We have $K\left(\phi_{n}\right) \leqslant K\left(\phi_{0}\right)$ for all $n$, and all $\phi_{n}$ are in the same isotopy class, so after passing to a subsequence we can construct a limiting combinatorial equivalence

$$
\left(\alpha_{0}, \alpha_{1}\right)=\lim \left(\phi_{n}, \phi_{n+1}\right)
$$

Here we use (A.1) to obtain, along a further subsequence, a bounded isotopy $\alpha_{t}=\lim \phi_{t+n}$ connecting $\alpha_{0}$ to $\alpha_{1}$.

Let $\Omega=\operatorname{int} \bigcap X_{n}$. Then $\alpha_{0}(x)=\alpha_{1}(x)$ outside $\Omega$. Moreover, $\alpha_{t}$ stabilizes $\Omega$ and $\alpha_{t} \mid \Omega$ is an isotopy rel ideal boundary. On each component of $\Omega$, replace $\alpha_{0}$ with the unique Douady-Earle map in the same isotopy class. (This map is obtained by lifting to the universal covers, and applying the Douady-Earle extension to the boundary values on $S^{1}$.) Since $f: \Omega \rightarrow \Omega$ is a holomorphic covering and the Douady-Earle extension is natural, these replacements are compatible with the dynamics and the resulting map $\psi$ is a quasiconformal conjugacy.

Remark. The dilatation of the conjugacy $\psi$ is bounded in terms of $K\left(\phi_{0}\right)$. The only increase in dilatation comes by applying the Douady-Earle extension.

Examples. (1) Let $f$ be a critically finite rational map on the sphere. Then $f: X_{1} \rightarrow X_{0}$ is a covering map, where $X_{0}=\widehat{\mathbf{C}}-P(f)$ and $X_{1}=f^{-1}\left(X_{0}\right)$. In this setting, the notion 
combinatorial equivalence was introduced by Thurston, who proved that combinatorially equivalent rational maps are conformally conjugate, apart from certain Lattès examples [DH2], [Mc3, §B].

(2) A similar principle holds when $P(f)$ is infinite. Namely, given a pair of isotopic quasiconformal maps $\phi_{i}:(\widehat{\mathbf{C}}, P(f)) \rightarrow(\widehat{\mathbf{C}}, P(g)), i=1,2$, with $g \circ \phi_{1}=\phi_{0} \circ f$, there is a quasiconformal conjugacy in the same isotopy class. This principle is used by Sullivan in his proof of rigidity of the Feigenbaum polynomial [Su].

(3) Consider a holomorphic family $f_{\lambda}(z)$ of rational maps such that the critical orbit relations are locally constant. By the theory of holomorphic motions, any two members of the family are combinatorially equivalent. Then they are also quasiconformally conjugate, by the theorem. Compare [McS, Theorem 7.1].

(4) If $X_{0}$ is connected and $\overline{X_{1}}$ is a proper compact subset of $X_{0}$, then $f: X_{1} \rightarrow X_{0}$ is an expanding conformal dynamical system. That is, $\left\|f^{\prime}\right\|>c>1$ in the hyperbolic metric on $X_{0}$. This expansion implies, for example, that $K(f)=\bigcap f^{-n}\left(X_{0}\right)$ is a compact nowhere dense set of Hausdorff dimension $<2$. The theorem provides structural stability for these mappings, since it is easy to construct a combinatorial equivalence from $f$ to a small perturbation $g$ of $f$.

(5) The mapping $f(z)=z^{2}+\lambda / z^{3}$, where $\lambda$ is small but not zero, is described in [Mc2]. For a suitable annulus $A$ centered at zero, we have $f^{-1}(A)=I \sqcup O \subset A$, where $I$ and $O$ are annuli covering $A$ with degree 3 and 2 respectively. It is easy to construct a combinatorial equivalence from $f:(I \sqcup O) \rightarrow A$ to $g:\left(I^{\prime} \sqcup O^{\prime}\right) \rightarrow A^{\prime}$, where $I^{\prime}, O^{\prime}$ and $A^{\prime}$ are round annuli centered at $z=0, g(z)=z^{2}$ in $O^{\prime}$ and $g(z)=\lambda / z^{3}$ in $I^{\prime}$. Clearly $K(g)$ is a Cantor set of round circles, so $K(f)$ is a Cantor set of quasicircles.

A rather different approach to this example appears in [Be].

A prototype of Theorem A.1 appears in [Mc1, Proposition 8.1]. 


\section{References}

[Ah1] AhLFors, L., Lectures on Quasiconformal Mappings. Van Nostrand, Toronto-New YorkLondon, 1966.

[Ah2] - Conformal Invariants: Topics in Geometric Function Theory. McGraw-Hill, New York-Düsseldorf-Johannesburg, 1973.

[As] Astala, K., Area distortion of quasiconformal mappings. Acta Math., 173 (1994), 37-60.

[Be] Beardon, A., Iteration of Rational Functions. Graduate Texts in Math., 132. SpringerVerlag, New York, 1991.

[D] Doundy, A., Disques de Siegel et anneaux de Herman, in Séminaire Bourbaki, vol. 1986/87, exp. $\mathrm{n}^{\circ}$ 677. Astérisque, 152-153 (1987), 151-172.

[DE] DouAdy, A. \& EARLE, C. J., Conformally natural extension of homeomorphisms of the circle. Acta Math., 157 (1986), 23-48.

[DH1] Douady, A. \& Hubbard, J., On the dynamics of polynomial-like mappings. Ann. Sci. École Norm. Sup., 18 (1985), 287-344.

[DH2] - A proof of Thurston's topological characterization of rational maps. Acta Math., 171 (1993), 263-297.

[EMc] Earle, C. J. \& MCMullen, C., Quasiconformal isotopies, in Holomorphic Functions and Moduli, Vol. I (Berkeley, CA, 1986), pp. 143-154. Math. Sci. Res. Inst. Publ., 10. Springer-Verlag, New York-Berlin, 1988.

[F] FARIA, E. DE, Asymptotic rigidity of scaling ratios for critical circle mappings. Preprint.

[FM] FARIA, E. DE \& MELO, W. DE, Rigidity of critical circle mappings. In preparation.

[He] Herman, M., Conjugaison quasi-symétrique des difféomorphismes du cercle et applications aux disques singuliers de Siegel. Manuscript, 1986.

[HW] Hardy, G. H. \& Wright, E. M., An Introduction to the Theory of Numbers, 5th edition. Oxford Univ. Press, New York, 1979.

[LV] Lehto, O. \& Virtanen, K. I., Quasiconformal Mappings in the Plane, 2nd edition. Grundlehren Math. Wiss., 126. Springer-Verlag, New York-Heidelberg, 1973.

[Mc1] MCMulles, C., Families of rational maps and iterative root-finding algorithms. Ann. of Math., 125 (1987), 467-493.

[Mc2] - Automorphisms of rational maps, in Holomorphic Functions and Moduli, Vol. I (Berkeley, CA, 1986), pp. 31-60. Math. Sci. Res. Inst. Publ., 10. Springer-Verlag, New York-Berlin, 1988.

[Mc3] - Complex Dynamics and Renormalization. Ann. of Math. Stud., 135. Princeton Univ. Press, Princeton, NJ, 1994.

[Mc4] - Renormalization and 3-Manifolds which Fiber over the Circle. Ann. of Math. Stud., 142. Princeton Univ. Press, Princeton, NJ, 1996.

[McS] McMullen, C. \& Sullivan, D., Quasiconformal homeomorphisms and dynamics III: The Teichmüller space of a holomorphic dynamical system. To appear in Adv. Math., 1998.

[MN] Manton, N.S. \& NauenberG, M., Universal scaling behavior for iterated maps in the complex plane. Comm. Math. Phys., 89 (1983), 555-570.

[Pe] Petersen, C. L., Local connectivity of some Julia sets containing a circle with an irrational rotation. Acta Math., 177 (1996), 163-224.

[Sh] Shishikura, M., The Hausdorff dimension of the boundary of the Mandelbrot set and Julia sets. SUNY Preprint, 1991/7.

[Si] Siegel, C. L., Iteration of analytic functions. Ann. of Math., 43 (1942), 607-612.

[St] Stein, E. M., Singular Integrals and Differentiability Properties of Functions. Princeton Math. Ser., 30. Princeton Univ. Press, Princeton, NJ, 1970. 
[Su] Sullivan, D., Bounds, quadratic differentials, and renormalization conjectures, in American Mathematical Society Centennial Publications, Vol. II (Providence, RI, 1988), pp. 417-466. Amer. Math. Soc., Providence, RI, 1992.

[Sw] ŚwiA̧teK, G., On critical circle homeomorphisms. Preprint, 1997.

[Wi] WIDOM, M., Renormalization group analysis of quasiperiodicity in analytic maps. Comm. Math. Phys., 92 (1983), 121-136.

\section{Curtis T. McMullen}

Department of Mathematics

Harvard University

1 Oxford Street

Cambridge, MA 02138

U.S.A.

ctm@math.harvard.edu

Received November 20, 1995

Received in revised form July 8, 1997 